\title{
Upcoming candidate cerebrospinal fluid biomarkers of Alzheimer's disease
}

Dementia due to Alzheimer's disease (AD) is estimated to reach epidemic proportions by the year 2030. Given the limited accuracy of current $A D$ clinical diagnosis, biomarkers of AD pathologies are currently being sought. Reductions in cerebrospinal fluid levels of $\beta$-amyloid 42 (a marker of amyloid plaques) and elevations in tau species (markers of neurofibrillary tangles and/or neurodegeneration) are well-established as biomarkers useful for AD diagnosis and prognosis. However, novel markers for other features of AD pathophysiology (e.g., $\beta$-amyloid processing, neuroinflammation and neuronal stress/dysfunction) and for other non-AD dementias are required to improve the accuracy of $A D$ disease diagnosis, prognosis, staging and therapeutic monitoring (theragnosis). This article discusses the potential of several promising novel cerebrospinal fluid analytes, highlights the next steps critical for advancement in the field, and provides a prediction on how the field may evolve in 5-10 years.

\section{KEYWORDS: Alzheimer's disease amyloid biomarker cerebrospinal fluid diagnostic accuracy neurodegeneration neurofibrillary tangles neuroinflammation prognosis theragnosis}

\section{The crisis of Alzheimer's disease}

Alzheimer's disease (AD), the most common cause of dementia in the elderly, is a progressive and fatal neurodegenerative disorder that currently affects approximately 10.6 million people in the USA and Europe, with projected estimates reaching epidemic proportions (15.4 million) by the year 2030 [201]. Alzheimer's disease leads to a loss of memory, cognitive function and, ultimately, independence, causing a heavy personal toll on the patients and their families and a tremendous financial burden on healthcare systems globally. Indeed, the cost for care of AD patients in 2011 in the USA alone was over US $\$ 183$ billion, with projected annual costs increasing to US $\$ 1$ trillion by the year 2050 unless effective disease-modifying treatments are developed [1].

A definitive diagnosis of $\mathrm{AD}$ requires postmortem identification of the presence of two hallmark brain lesions - extracellular deposits of the $\beta$-amyloid $(A \beta)$ peptide (amyloid plaques) and intraneuronal accumulations of hyperphosphorylated tau protein (neurofibrillary tangles). A clinical diagnosis of AD during life (dementia of the Alzheimer type [DAT]) is based on guidelines established by the National Institute of Neurological Disorders and Stroke-Alzheimer's Disease and Related Disorders Association (NINCDS-ADRDA) [2]. Unfortunately, the accuracy of current clinical AD diagnostic methods to predict pathologic diagnoses (in the absence of biomarker information), although promising in some centers, is generally quite low; a recent study involving research participants $(\mathrm{n}>900)$ evaluated in more than 30 Alzheimer's Disease Centers in the USA reports sensitivities ranging from 70.9 to $87.3 \%$ and specificities from 44.3 to $70.8 \%$ (depending on the specific histopathological diagnostic criteria employed) [3]. This variable and relatively poor performance is particularly troubling given the high level of expertise of the clinicians in such specialized AD centers. Diagnostic accuracies in secondary or primary care settings are likely even lower. Therefore, there is an urgent need for objective tests that can increase diagnostic accuracy in the shorter term, to aid in the design and evaluation of treatment efficacy of clinical trials, and in the longer term, for individual patient care.

Another imperative is early diagnosis. Clinical trials of AD therapeutics to date have been largely unsuccessful in reversing cognitive decline. Growing consensus attributes at least some of this failure to the exclusive enrollment of individuals who already exhibit mild or moderate dementia. At even earlier stages of the disease (very mild dementia), neuronal losses in certain critical brain regions are already severe [4]. Thus, it is critical to diagnose individuals at very early disease stages - and enroll them in clinical trials - in order to identify and apply therapies with the best chance of preserving normal cognitive function.

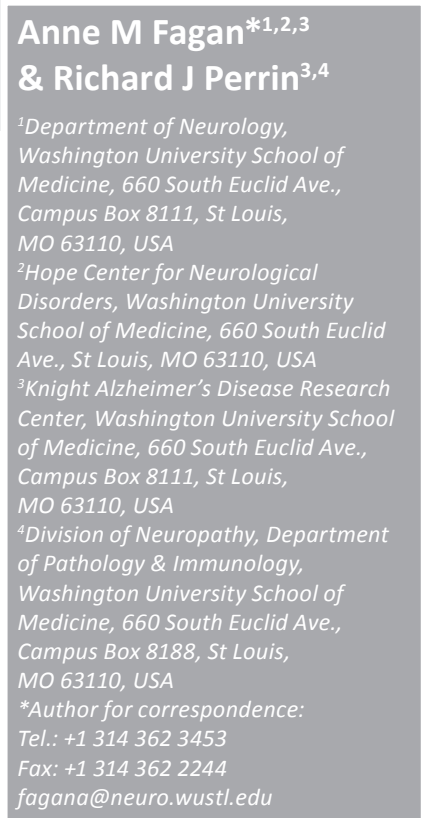

Future
Medicine
part of 


\section{Alzheimer's disease is a chronic disease}

The past several years have brought about a renewed appreciation of the chronic, evolving nature of AD pathogenesis. The clinical construct of mild cognitive impairment (MCI) [5], defined by impairments in cognitive abilities (compared with age-matched normative values) that are below the threshold considered to be 'dementia' has been hypothesized to represent a transition between healthy aging and DAT for those with AD pathology. Furthermore, clinicopathological correlation studies support the notion of a long asymptomatic ('preclinical') stage of the disease, with brain pathology estimated to begin years, even decades, prior to significant neuronal cell death and the appearance of any behavioral signs or symptoms, including MCI [4,6-10]. This appreciation has fueled a paradigm shift in therapeutic goals from disease 'cure' (considered to be virtually impossible in dementia stages associated with significant neuron loss), to halting, delaying or even preventing cognitive decline due to AD pathology in the very early stages of the disease.

\section{Established cerebrospinal fluid biomarkers of $A D$}

Given the current limitations of clinical diagnostic accuracy during the preclinical and early clinical stages of the disease, fluid (and imaging) biomarkers of AD pathologies are currently being sought. A biomarker is a characteristic that is objectively measured and evaluated as an indicator of normal biological processes, pathogenic processes or pharmacologic responses to a therapeutic intervention [11]. As such, a biomarker can be used to guide clinical diagnosis (diagnostic), estimate disease risk or prognosis (prognostic), evaluate disease stage (staging) and monitor progression and/or response to therapy (theragnostic) [12].

Three proteins in the cerebrospinal fluid (CSF) have been well-established internationally as biomarkers of $\mathrm{AD}-\mathrm{A} \beta 42$, total tau and phosphorylated tau181 (p-tau181). In general, individuals with AD exhibit low levels of CSF A $\beta 42$ and high levels of total tau and p-tau181 compared with cognitively normal, age-matched controls [12-14]. Low levels of CSF A $\beta 42$ are associated with cortical amyloid as evidenced by retention of the amyloid-binding agent, Pittsburgh compound B (PiB), detected by PET [15-19]. CSF levels of total tau positively correlate with the amount of tissue damage and poor clinical outcome in acute brain disorders [20,21], suggesting it reflects the intensity of neuronal degeneration. Some hyperphosphorylated forms of tau measured in CSF samples obtained during life have been shown to correlate with the amount of neocortical tangle pathology at autopsy [22] suggesting some forms of CSF p-tau may serve as markers of tangle pathology. Elevated tau and p-tau181 on their own have been shown to predict progression from MCI to $\mathrm{AD}$ in some [23] (especially when combined with $A P O E$ genotype) but not all $[24,25]$ studies. However, the ratio of $\operatorname{tau}(\mathrm{s})$ to $A \beta 42$ has been shown by several groups to be highly predictive of cognitive decline in cognitively normal cohorts [25-27] as well as individuals with MCI or very mild dementia [24,27-31]. Characterizing the precise timing of $\mathrm{AD}$-related biomarker trajectories over the extended course of the disease is the subject of many current research efforts, the results of which will have practical implications for the design of clinical trials and, eventually, disease-modifying therapy. In support of this idea, a recent meta-analysis suggests that use of baseline levels of CSF A $\beta 42$ and tau to select MCI cohorts with AD pathology in a fictitious clinical trial would reduce the needed sample size by $67 \%$ and trial costs by $60 \%$ compared with a trial in which unselected MCI participants would be enrolled [32].

\section{The need for novel biomarkers}

Despite the association between the levels of the more established CSF analytes and underlying AD pathology, measures of biomarker accuracy for clinical diagnosis vary widely between studies. Such variability also underscores the need for the development of additional biomarkers that will, either on their own or in combination with more established markers, increase diagnostic accuracy. Novel biomarkers are also needed that will identify additional processes involved in AD pathogenesis, such as markers of neuroinflammation and early neuronal stress and dysfunction prior to overt cell death. Given that other neurodegenerative conditions (e.g., dementia with Lewy bodies [DLB], frontotemporal lobar degeneration [FTLD], vascular dementia [VaD], progressive supranuclear palsy [PSP] and hippocampal sclerosis) can present with AD-like clinical symptoms, and individuals with AD frequently have comorbid pathologies, additional markers are needed that can aid in differential diagnosis and identify mixed pathologies. Markers will also be required to define pathophysiological stages of AD (in parallel with clinical stages; preclinical, 
MCI, DAT) and identify those individuals at increased risk of rapid cognitive decline.

Cohort uniformity will likely improve clinical trial design and interpretation, and preferential enrollment of individuals with relatively precipitous trajectories may shorten clinical trial duration and increase the power of cognitive outcome measures. Future treatments may target stage-specific mechanisms, and responses to these treatments may differ as a function of disease stage. Finally, while treatments are being evaluated for effect, pathological stage-defining biomarkers should allow evaluation of efficacy, in addition to or instead of cognitive/behavioral measures.

\section{Upcoming candidate CSF biomarkers}

The pathophysiology of AD is complex, involving amyloid plaque deposition and evolution, gliosis/neuroinflammation, neurofibrillary tangle formation and synaptic and neuronal loss, and the composition of CSF undergoes changes that reflect this complexity. Consequently, many molecules have been identified as candidate AD biomarkers (TAвlE 1). Since the sophistication and power of proteomics technology has evolved tremendously over the last decade, proteins comprise the vast majority of viable candidates. Individually, many candidates show utility for measuring statistical differences between cohorts of $\mathrm{AD}$ and 'control' samples, and many of these also show potential to improve the diagnostic accuracy of $A \beta 42$, tau and p-tau. A shorter list of candidates shows potential to track disease progression, and even fewer have been reported to predict the rate of cognitive decline.

The goal of the present review is to discuss current promising CSF analytes beyond the well-established markers, $A \beta 42$, total tau and p-tau. Efforts have involved defined, hypothesis-driven approaches (e.g., specified molecules involved in $A \beta$ metabolism, neurodegeneration, oxidative stress and neuroinflammation, among others), as well as unbiased and targeted multi-analyte profiling strategies (e.g., proteomics screens and use of defined molecular arrays). This review will focus on some of the more promising biomarkers that appear to have utility for one or more of the following: diagnosis/differential diagnosis; prognosis; and monitoring/staging/theragnosis. Direct comparison of the potential utility of the various candidate markers is not practical due to differences in the cohorts evaluated and the methodological and analytical procedures employed. Furthermore, these lists should not be regarded as exclusive; based on our review of the literature, the markers included can be considered promising enough to warrant further investigation in larger, well-characterized cohorts. Among the leading candidates are some that are believed to be related to $A \beta$ generation, metabolism and/or amyloidogenesis.

\section{APP processing sAPP- $\beta$}

The A $\beta$ peptide, the primary constituent of amyloid plaques, is generated through serial cleavage of APP by two secretases termed $\beta$-secretase and $\gamma$-secretase (Figure 1) [33]. Upon cleavage by $\beta$-secretase (i.e., BACE1, see below), the large $\mathrm{N}$-terminal domain of APP, termed sAPP- $\beta$, is released as a soluble form into brain interstitial fluid and eventually reaches the CSF. A $\beta$ (in several amino acid lengths) is released through subsequent intramembranous cleavage of the APP- $\beta$ C-terminal fragment by $\gamma$-secretase. Levels of CSF sAPP- $\beta$ have been reported to be unaltered or mildly elevated in sporadic AD and MCI [34-38]. When combined with CSF tau, sAPP- $\beta$ has been shown to be useful in predicting cognitive decline in MCI cohorts $[39,40]$.

\section{BACE1}

The primary secretase responsible for the cleavage of $A P P$ at the $\beta$-site to produce $A \beta$ is BACE1 [41]. BACE1 levels and activity in CSF have been reported to be increased in clinical and prodromal AD [37,42-46], making it a promising biomarker candidate. However, it may have limited utility for individual diagnosis/prognosis due to substantial overlap between levels in AD cases and controls.

\section{SAPP- $\alpha$}

APP is a substrate for another proteolytic complex, $\alpha$-secretase, which liberates a different fragment, termed sAPP- $\alpha$. Since $\alpha$-secretase cleaves APP within the A $\beta$ sequence itself, preventing the generation of $A \beta$, this pathway is considered to be nonamyloidogenic (Figure 1). Similar to sAPP- $\beta$, levels of sAPP $\alpha$ in the CSF have been reported to be unaltered or mildly elevated in sporadic AD and MCI [34-37]. Because these published data remain inconclusive, sAPP- $\alpha$ and sAPP- $\beta$ do not top the list of promising diagnostic or prognostic biomarkers. However, they may be particularly useful as theragnostic markers in clinical trials designed to alter APP processing. This possibility awaits investigation. 


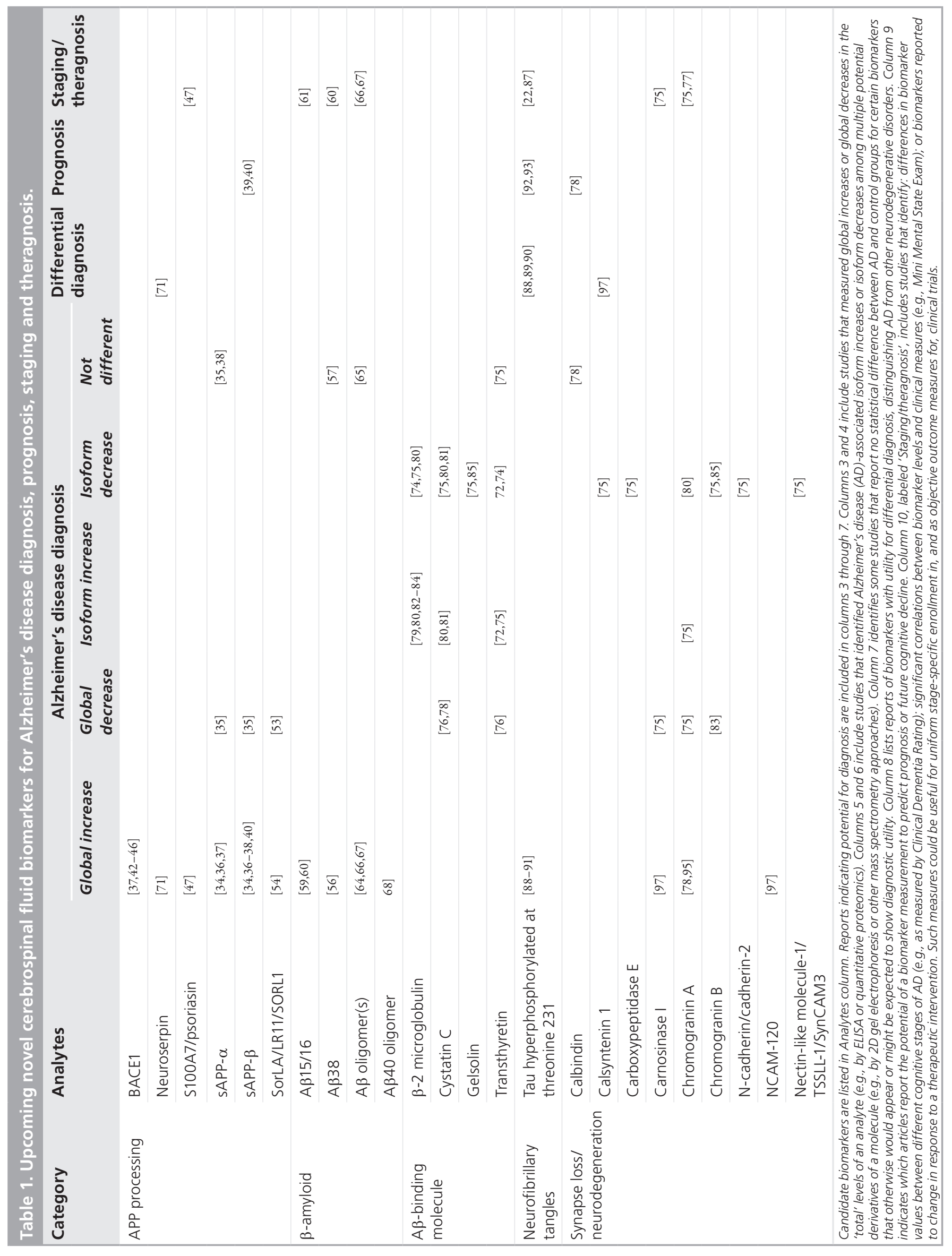




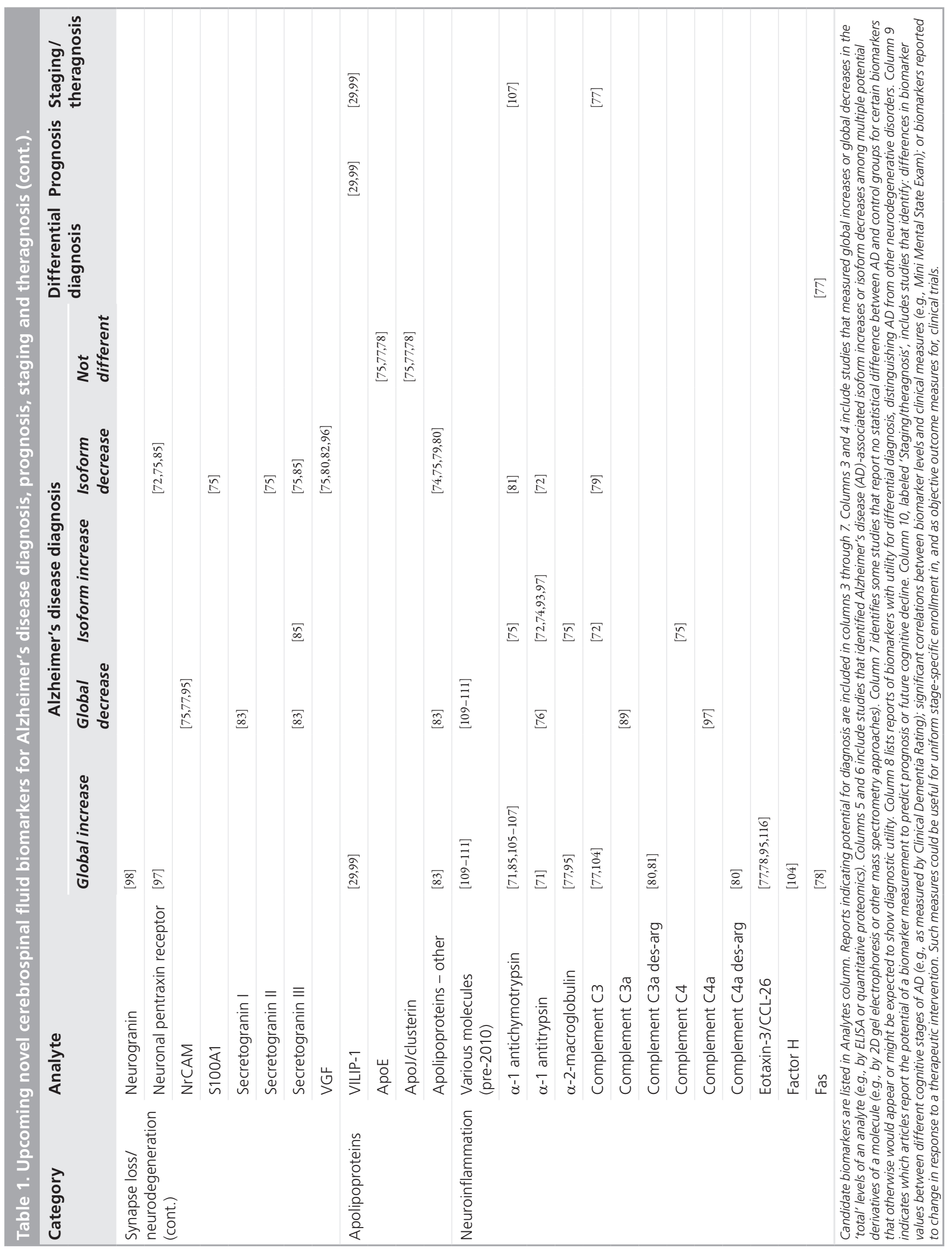




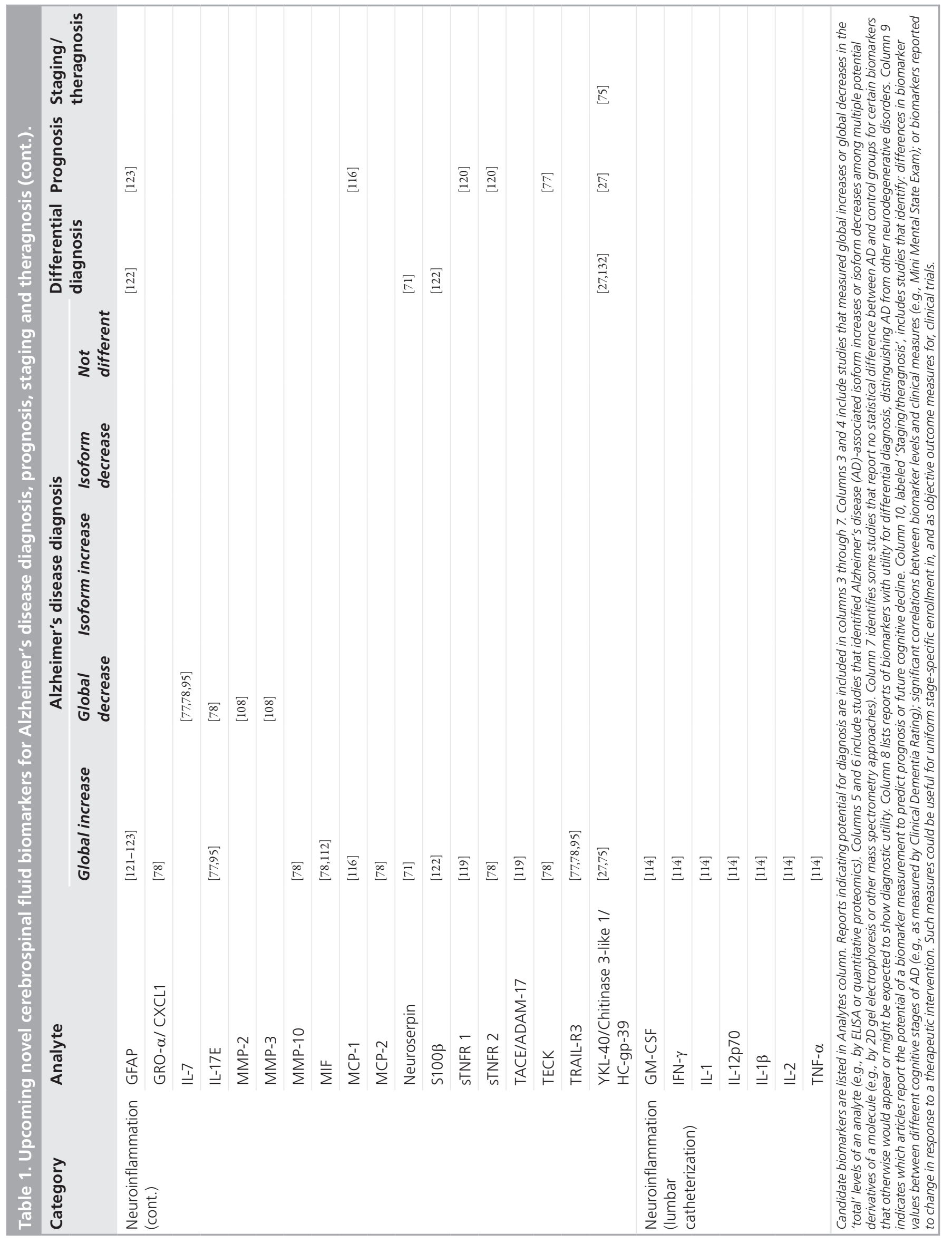




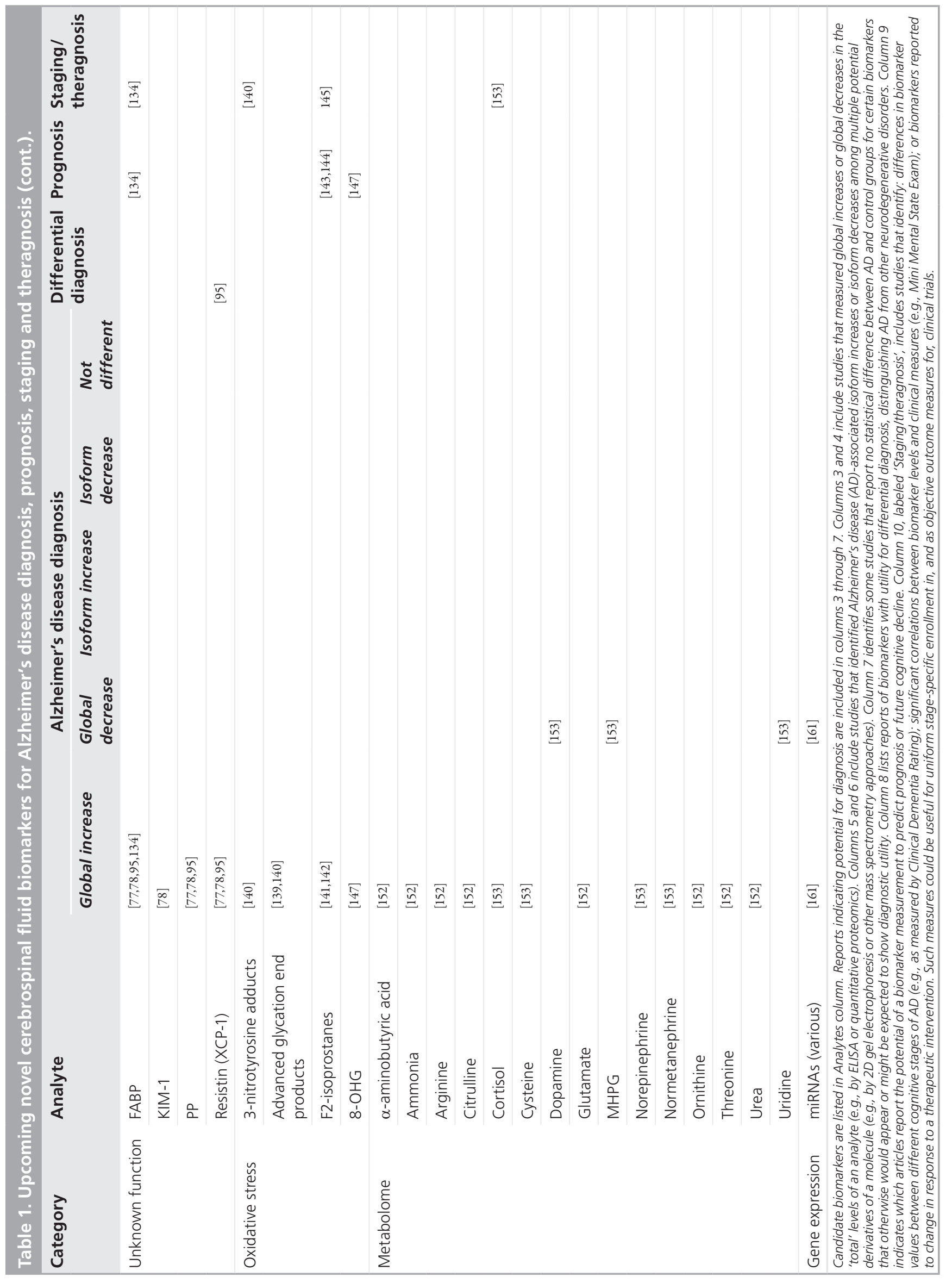




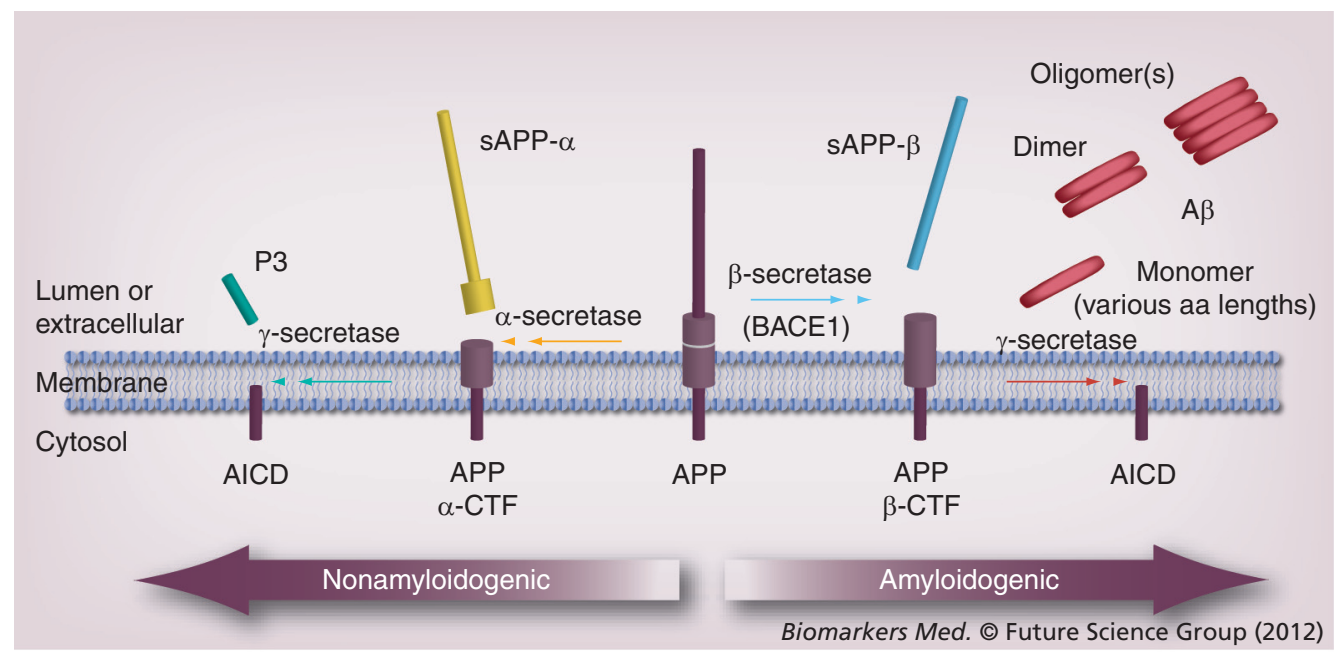

Figure 1. APP processing in the amyloidogenic and nonamyloidogenic pathways. APP is a type I transmembrane protein that is the substrate for several cleaving enzyme complexes known as secretases. In the amyloidogenic pathway (right), APP is cleaved at the $\beta$-secretase site by BACE1, releasing a large $\mathrm{N}$-terminal domain of APP (termed SAPP- $\beta$ ). The remaining membrane-bound CTF (termed APP $\beta$-CTF) undergoes subsequent intramembranous cleavage by the $\gamma$-secretase complex to release the $A \beta$ peptide (of various numbers of amino acids in length). Secreted $A \beta$ exists in monomeric, dimeric and likely several oligomeric forms. The remaining membrane-associated fragment $(A I C D)$ has been shown to translocate to the nucleus where it exerts signaling functions. In the nonamyloidogenic pathway (left), APP is cleaved at the $\alpha$-secretase site within the A $\beta$ sequence itself by another proteolytic complex, releasing a slightly longer $\mathrm{N}$-terminal domain of APP (termed SAPP- $\alpha$ ). The remaining membrane-bound CTF (termed APP $\alpha$-CTF) undergoes subsequent intramembranous cleavage by the $\gamma$-secretase complex, releasing a nonamyloidigenic fragment of the $A \beta$ peptide, termed P3. Not pictured, yet germane to the topic of upcoming CSF biomarkers of $A D$, is SorLA/sLR11, a neuronal receptor for APP that controls its intracellular transport and processing, blocking both the amyloidogenic and nonamyloidogenic pathways, and S100A7/psoriasin, a calcium-binding protein shown to promote $\alpha$-secretase cleavage via effects on ADAM-10, an enzyme with known $\alpha$-secretase activity.

A $\beta$ : $\beta$-amyloid; aa: Amino acid; AICD: APP intracellular domain; CTF: C-terminal fragment.

\section{S100A7}

S100A7, also known as psoriasin, has been reported in a proteomics study to be elevated in AD CSF compared with control [47]. S100A7 has been previously implicated in inflammatory responses and cell differentiation, among other functions [48], but its role in the normal or diseased brain is unknown. Interestingly, S100A7 may be involved in AD by influencing APP processing. S100A7 has been shown to promote $\alpha$-secretase cleavage by increasing the activity of ADAM-10, an enzyme with $\alpha$-secretase activity [47]. Additional study of this potential marker is warranted.

\section{SorLA/sLR11}

The sorting protein-related receptor with A-type repeats (SorLA; also known as SorL1 or LR11) has been implicated in AD. SorLA/LR11 is a neuronal receptor for APP that controls its intracellular transport and processing, blocking both amyloidogenic and nonamyloidogenic processing of APP [49]. SorLA is downregulated in AD brain [50,51], and SORL1 gene variants have been shown to be an $\mathrm{AD}$ genetic risk factor [52]. Soluble forms of SorLA (sLR11) are found in CSF, with levels (detected by western blotting) reported to be decreased in a small number of $A D$ patients [53]. A more recent, larger study utilizing quantitative ELISA reported statistically significant elevations in AD CSF [54], but the increase was relatively small, and the diagnostic value for individual patients was limited. Validation in larger, well-characterized cohorts is needed.

\section{$\beta$-amyloid}

\section{Truncated $A \beta$ species}

Many studies have investigated CSF A $\beta 40$ (40 amino acids in length, the most abundant isoform in CSF) and $\mathrm{A} \beta 42$ as biomarkers of $\mathrm{AD}$, but several C-terminally truncated species of $A \beta$ have also been evaluated (TaвLE 1). Unlike CSF $\mathrm{A} \beta 42$, which is markedly decreased in the setting of cortical amyloid deposits as determined by amyloid imaging [55], CSF A $\beta 38$ has been reported to be increased or not changed in $\mathrm{AD}$ $[56,57]$. However, levels of CSF A 338 do not correlate with the presence of cortical amyloid [55], 
thus casting doubt upon its potential to serve a similar role as an antecedent (or preclinical) biomarker. Several C-terminally truncated A $\beta$ isoforms have also been identified in CSF [58], and levels of $A \beta 16$ have been reported to be higher in AD compared with controls [59,60]. Furthermore, levels of $A \beta 16$ increase in response to treatment with a $\gamma$-secretase inhibitor [61], supporting its potential use as a theragnostic marker in clinical trials targeting $A \beta$ production.

\section{$A \beta$ oligomers}

Although parenchymal deposits of insoluble $A \beta$ represent a defining pathologic feature of $\mathrm{AD}$, results from cell culture and animal studies suggest an important role for soluble $A \beta$ oligomers in $\mathrm{AD}$ pathogenesis [62]. In spite of this growing body of evidence, consensus about the relevant oligomeric species (e.g., dimer, trimer, tetramer, 12-mer $\left[A \beta 56^{*}\right], A \beta$-derived diffusible ligands, high-molecular-weight species) is lacking; experimental preparations of $A \beta$ oligomers have been highly variable and the methods employed to characterize such preparations remain diverse, often imprecise, and controversial [63]. Furthermore, quantifying and even proving the existence of $A \beta$ oligomers in CSF have been difficult. Studies have employed various methods/ strategies, including flow cytometry, immunoprecipitation-western blotting (IP-WB), ELISA and aggregate capture assays. One study using nanoparticle-based detection methods reported increases in oligomeric $\mathrm{A} \beta$ species in AD CSF samples [64], while another study utilizing IP-WB found no clear correlation with clinical disease [65]. Two more recent studies, one utilizing an ELISA that recognizes high-molecular-weight $(40-200 \mathrm{kDa})$ oligomers of $A \beta[66]$, and the other using flow cytometry [67], reported elevations in $\mathrm{AD}$ patients compared with controls and positive correlations with the degree of cognitive impairment $[66,67]$. In another study using a novel assay (misfolded protein assay) designed to capture and detect aggregates of specific $A \beta$ species, AD CSF was found to contain higher levels of oligomers of $A \beta 40$ (but not $A \beta 42$ ) compared with control CSF [68]. Overall these results are indeed promising. However, each of the different assays awaits verification of the true nature of the oligomeric species being measured, and each of these results awaits validation in larger independent cohorts.

\section{Neuroserpin}

Neuroserpin (NS), a serine protease inhibitor, is associated with amyloid plaques in the $\mathrm{AD}$ brain [69,70], and levels are elevated in AD (but not DLB) CSF compared with controls [71]. Neuroserpin has been implicated in $A \beta$ metabolism [69,70], but it serves many functions (e.g., neuroinflammatory), some of which could conceivably impact AD pathogenesis independent of (or in addition to) its effects on A $\beta$. Regardless of the mechanism(s), NS is a candidate AD CSF biomarker that warrants further investigation.

\section{- $A \beta$-binding proteins}

Just as $A \beta$ oligomerization and deposition are important to the initiation of AD pathology, so, too, are the proteins that function to prevent these processes. Indeed, many proteins that have been characterized as $A \beta$-binding proteins have appeared as promising biomarkers (TABLE 1).

\section{Transthyretin}

Transthyretin has a storied past as an AD CSF biomarker, in part because it is so abundant, but also because of its molecular complexity. This product of the choroid plexus has been identified in the majority of proteomics studies that are sensitive to isoform differences [72-75], and by immunoassay in one small study [76]. Larger studies applying bead- or plate-based ELISA formats have detected no statistical differences $[75,77,78]$ likely due to the inability of these assays to discriminate the different isoforms. Transthyretin in CSF also appears to fluctuate substantially within a single individual over a 2-week interval [79], casting some doubt as to its potential as a disease biomarker.

\section{Cystatin C}

Measurements of AD-related changes in CSF cystatin $\mathrm{C}$ by immunoassay have produced mixed results $[75,76,78]$. These variable findings may be related to the apparent delayed decline of this protein with disease progression that would be missed in AD cohorts with mixed severities [75]. In one large study of individuals diagnosed with very mild and mild DAT, cystatin C complemented the tau: $A \beta 42$ ratio in distinguishing AD from cognitively normal controls despite its lackluster performance as a marker on its own [78]. In the future, assays to target a C-terminally truncated form of cystatin $\mathrm{C}$ that is increased in AD CSF $[80,81]$ may be worth pursuing.

\section{$\beta-2$ microglobulin}

$\beta-2$ microglobulin is another $A \beta$-binding protein that has appeared in many isoform-sensitive proteomics studies [73-75,79,80,82-84], but has failed to show utility as measured by several different immunoassays [75,77,78]. 


\section{Gelsolin}

Gelsolin is an abundant $A \beta$-binding protein that has also been identified as a candidate diagnostic biomarker in proteomics studies, but has not been validated by subsequent immunoassays $[75,85]$. This failure may be due to assay insensitivity to different gelsolin isoforms [85], but may also reflect post-translational modifications that have been described in $\mathrm{AD}$ brain tissue [86].

\section{Neurofibrillary tangles}

Although the majority of AD studies have evaluated levels of p-tau181 as a potential biomarker, tau hyperphosphorylated at threonine 231 has been reported to correlate with tangle load better than p-tau181 (TABLE 1) [22,87]. Levels are elevated in $\mathrm{AD}$ compared with controls and may also show greater utility for distinguishing $\mathrm{AD}$ from other disorders including geriatric depression, DLB, VaD and FTLD [88-90]. Elevated levels of p-tau231 are also observed at earlier MCI stages [91] and predict future cognitive decline $[92,93]$. Various truncated forms of tau have also been reported in AD brain [94] and conceivably could prove to be useful disease biomarkers if found to be present and measurable in CSF. This possibility awaits investigation.

\section{- Synapse loss/neurodegeneration}

Beyond CSF markers associated with amyloid processing/deposition and neurofibrillary tangles, many putative indicators of synapse loss and neuronal injury/degeneration (in addition to total tau) have emerged as promising candidates (Table 1). For example, the dense core vesicle protein chromogranin A has been identified in many studies, both in its intact form measured by immunoassay $[75,77,78,95]$, and as proteolytic fragments [80,96]. Additional dense core protein family members such as VGF $[75,80,82,96]$, carboxypeptidase E, chromogranin B, secretogranin I, secretogranin II and secretogranin III have also been identified in proteomics screens $[75,83,85]$. Levels of synaptic adhesion molecules such as NrCAM [75,77,95], NCAM-120 [97], neuronal pentraxin receptor $[72,75,85,97]$, calsyntenin 1 [75,97], N-cadherin and nectin-like molecule-1/ TSSLL-1/SynCam3 [75] have also been widely reported to change in $\mathrm{AD}$, as have levels of the post-synaptic protein neurogranin [98] and neuronal proteins carnosinase I $[75,85,97]$ and S100A1 [75]. Finally, considered to be indicators of neuronal damage, increased CSF VILIP-1 has shown utility for diagnosis [29,99], and both VILIP-1 $[29,99]$ and calbindin $[78]$, as ratios with $A \beta 42$, show utility comparable to that of the tau:A $\beta 42$ ratio for predicting cognitive decline [78].

\section{Apolipoproteins}

Despite the known role of apoE and apoJ/clusterin in $\mathrm{AD}$ pathogenesis $[100,101]$, it is perhaps surprising that levels of apoE and clusterin in CSF have shown little promise as biomarkers. CSF apoE concentrations vary according to $A P O E$ genotype among cognitively normal individuals, with the $\mathrm{AD}$-associated risk $\varepsilon 4$ allele associated with the highest, and the $\varepsilon 2$ allele with the lowest concentration [102]. However, it remains unclear whether a change in CSF apoE level can be attributed to underlying $\mathrm{AD}$ pathology; results have been inconsistent [100]. CSF levels of apoE and apoJ/ clusterin in larger proteomics screens have similarly failed to show a statistically significant difference between $\mathrm{AD}$ and healthy controls [75,77,78].

As assessed by immunoassays, the story for other apolipoproteins is no different, with the exception of apoD (Table 1). ApoD is reported to be increased in AD CSF $[78,95,103]$. As assessed by proteomics techniques that are more sensitive to isoform differences, many apolipoproteins (including ApoA-1, -CII, -CIII and -H) have shown AD-associated changes [72,74,75,79,80,83]. The potential significance of these more subtle changes is unclear. Further investigation in large, well-characterized cohorts is warranted.

\section{- Neuroinflammation}

Mechanistically implicated as both neurotoxins and neuroprotectants in $\mathrm{AD}$ pathophysiology, molecules of 'neuroinflammation' are also widely reported as candidate biomarkers (Table 1). This category, which encompasses products of microglia, astrocytes, neurons, blood vessels, and even other sources outside the CNS, is broad. Included are cytokines, chemokines, complement proteins, proteases, protease substrates and their cleavage products, protease inhibitors, and other glia-derived proteins with well-known, little-known or unknown functions in the brain. Many of these molecules have received preferential attention as potential biomarkers because they are abundant in CSF - thus, easily 'discovered' in proteomic screens - and/or robust commercially-available multiplexed assays are available for their measurement $[77,78,95]$.

\section{Complement proteins/proteases/ protease inhibitors}

Most candidate biomarkers in this category have been reported for their potential diagnostic utility, 
but some have additionally been proposed as markers to track progression. Some 'stand-outs,' several of which have been reported repeatedly, include complement proteins C3 [72,77,79,104], C3a [97], C3a des-arg [80,81], C4 [75], C4a [97], C4a des-arg [80], C3/C4 homologous protein $\alpha$-2-macroglobulin [75,77] and factor $\mathrm{H}$ [104]. In the category of protease-inhibiting serpins, $\alpha-1$ antitrypsin $[71,72,74,76,83,97], \alpha-1$ antichymotrypsin $[71,75,81,85,105-107]$ and neuroserpin [71] have received particular attention. $\alpha-1$ antichymotrypsin additionally shows potential for disease staging [107]. Complementing the serpins are matrix metalloproteinases MMP-2 and MMP-3, which appear to correlate with A $\beta 42$ in CSF [108], and MMP-10, which appears to be increased in AD [78].

\section{Cytokines}

Although cytokines have received more attention in blood-derived fluids (serum, plasma) than in CSF, scores of AD-related CSF studies have been reported before 2010 (reviewed in [109-111]). Unfortunately, these studies achieved little consensus. Since then, larger studies with somewhat greater congruence have been reported $[77,78,95]$. Currently, in our estimation, CSF biomarkers for AD in this category that show greatest consensus include: reduced levels of IL-7 and IL-17E [77,78,95] and increased macrophage MIF, which induces TNF- $\alpha$, IL-6 and IFN- $\gamma[78,112]$. It is worth noting, however, that the cytokine response to insult, rather than baseline CSF levels, might demonstrate more striking differences between AD and controls. An exaggerated cytokine response has been described in peripheral monocytes derived from individuals with AD compared with monocytes from healthy controls [113]. In a recent study, CSF levels of many cytokines (IL-1 $\beta$, IL-2, IL-10, IL-12p70, GM-CSF, IFN- $\gamma$ and TNF$\alpha$ ), but not IL- 6 or IL- 8 , were noted to increase dramatically in $\mathrm{AD}$ patients in response to intrathecal catheterization and repeated CSF draws over a 24-h period [114]. The mechanism of this differential response remains unclear, but is worthy of further investigation, particularly as indwelling intrathecal catheterization is being used in research settings [115] and will likely be used to evaluate 'target engagement' in response to therapy. Independent of how cytokine changes might impact concentrations of noncytokine molecules in CSF, the apparent volatility of cytokine levels in response to this relatively innocuous stimulus (or technique of sampling) may account, at least in part, for the variable results reported in different studies.

\section{Chemokines}

Closely related to the cytokines are the chemokines. These signaling molecules are similarly great in number, in attention received, and in mixed results (reviewed in [111]). They also have been examined recently in larger studies $[77,78,95]$. Leading candidates among these molecules include: eotaxin-3/CCL-26, which is increased in MCI [116], AD [77,78,95] and other neurological disorders $[95,117]$ relative to healthy controls; monocyte chemotactic proteins, which show promise for prognosis (MCP-1) [116] and diagnosis (MCP-2) [78]; thymus-expressed chemokine, which is increased in $\mathrm{AD}$ versus controls [78] and correlates with subsequent rate of cognitive decline in MCI [77]; and GRO- $\alpha$ /CXCL1, which appears to be expressed by microglial cells [118] and is increased in AD versus controls [78].

\section{Cytokine receptors}

Cytokine receptors are also closely related to cytokines, but in a different fashion. Several of these proteins are cleaved from the cell surface, yielding a soluble version that migrates from the brain into the CSF. CSF concentrations of soluble TNF- $\alpha$ receptors (sTNFRs) 1 and 2 are higher in AD compared with controls [78,119], and are elevated even further in individuals with MCI relative to those with AD dementia [119]. Moreover, among individuals with MCI, levels of sTNFRs are higher in those who progressed to AD compared with those who remain stable [120]. sTNFRs are liberated from the membrane by TACE/ADAM-17, and the activity of this enzyme is also greater in AD than controls [119]. However, these markers are not specific for ADsince elevated levels are also observed in $\mathrm{VaD}$ [120]. TRAIL-R3 is another marker that is increased in AD CSF [77,78,95]. As its acronym implies, TRAIL-R3 is a member of the TNF- $\alpha$ receptor family. How it is released into CSF, and whether its increase in AD reflects greater production within or greater depletion from the brain is not clear. Finally, levels of Fas, another member of the TNF- $\alpha$ receptor family, also differ in AD versus control CSF [78]. Although CSF Fas is also increased in other neurodegenerative disorders (e.g., FTLD [117]), it may be useful as part of a panel of markers assembled for distinguishing AD from other neurodegenerative diseases [77].

\section{Astrocytic proteins}

Perhaps more straightforward than the above peptides and receptors of neuroinflammation 
are the structural elements of astrocytes themselves. GFAP and S100 $\beta$, two molecules that are well-established as immunohistochemical and biochemical markers of astrocytosis, have also been reported as potential candidate CSF biomarkers for AD diagnosis [121-123]. CSF GFAP additionally appears to correlate with dementia severity [123]. Not surprisingly, because astrocytosis represents a fundamental reaction of the CNS to injury, these molecules are also increased in the setting of other neurological disorders and brain injuries (e.g., CreutzfeldtJakob disease [121,122], multiple sclerosis [124], stroke [125-127] and acute [128,129] and repetitive [130] traumatic brain injury). Nevertheless, like most novel biomarker candidates, GFAP and $S 100 \beta$ may show some utility as part of a biomarker panel, particularly for staging, prognosis or theragnosis. Indeed, even for differential diagnosis, these two markers together show some capacity to distinguish Creutzfeldt-Jakob disease from $\mathrm{AD}$ [122].

\section{Miscellaneous/unknown function Chitinase 3-like 1/YKL-40}

Although it is included here in the 'miscellaneous' category of AD CSF biomarkers, YKL-40/chitinase 3-like 1/HC-gp-39 serves as a reasonable segue from discussions of astrocytosis and TNF- $\alpha$ receptors because one of its proposed functions is to limit some of the effects of inflammatory cytokines [131]. Moreover, although YKL-40 is produced by monocyte-derived cells in the periphery, YKL-40 is produced in the brain not by microglial cells, but by astrocytes that are accompanied by activated microglia, likely producing TNF- $\alpha$, IL- $1 \beta$ and other factors $[27,132,133]$. In $A D$, the astrocytes in question appear to be a small subset of those associated with amyloid plaques. Like CSF tau, CSF YKL-40 is increased in the very early stages of $\mathrm{AD}$ (very mild dementia/MCI) and remains high at later stages [27,75]. Thus, particularly as part of a biomarker panel, YKL-40 may show utility for monitoring very early stages of AD pathology. As with CSF tau, levels of CSF YKL- 40 as a ratio with $A \beta 42$ can predict the rate of cognitive decline [27]. CSF YKL-40 shows some limited potential for distinguishing AD from other neurodegenerative diseases, such as PSP, but not FTLD [27] or stroke [132]. Evaluation of YKL-40 in DLB has not been reported.

\section{Heart FABP}

Heart FABP is another new, promising CSF biomarker that is not easily classified. Although its function in brain is not completely understood, a 'knockout' mouse model suggests an important role in arachidonic acid incorporation into phospholipids. Regardless of its function, CSF levels of FABP are elevated in $\mathrm{AD}[77,78,95,134]$ and in those with MCI who subsequently progress to $\mathrm{AD}$ [134]. Additionally, the FABP:A $\beta 42$ ratio appears to correlate with the rate of decline in the Mini Mental State Exam Score (MMSE) [134], and thus, like YKL-40/A $\beta 42$, may have prognostic utility. However, FABP may have limited potential for differential diagnosis $[95,117]$.

\section{Kidney injury molecule-1}

Even less understood in the context of brain physiology is KIM-1, which was identified as an AD biomarker only because it was targeted in a large multiplex immunoassay panel. Nevertheless, CSF KIM-1 shows utility for distinguishing AD from controls and complements the traditional markers, tau and $A \beta 42$, very effectively $[78]$.

\section{Pancreatic polypeptide/resistin}

These two candidate markers appear somewhat more relevant to emerging theories about $\mathrm{AD}$ pathogenesis in that they may have a role in regulating the metabolism of glucose $[135,136]$ and $A \beta$ [137]. Interestingly, neuroanatomic maps of greatest 'aerobic glycolysis' in the brain appear to overlap areas of maximal amyloid deposition in $\mathrm{AD}$ [138]. Pancreatic polypeptide (PP) and resistin (XCP-1) are increased in AD CSF [77,78,95], and resistin additionally appears to be specific for $\mathrm{AD}$, relative to non-AD neurodegenerative disorders [95].

\section{Oxidative stress}

In addition to markers associated with amyloid, tangles, neuronal damage, gliosis and neuroinflammation, several biomarkers with great potential are produced by more fundamental chemical reactions that accompany the histologically observable changes of AD. In particular, markers of pathological oxidative processes have received attention (TABLE $\mathbf{1}$ ).

\section{3-NT adducts/advanced glycation end products}

Proteins have a role in this area as carriers of 3-nitrotyrosine adducts (spawned by the attack of peroxynitrite on tyrosine residues) and advanced glycation end products. Such modified proteins in CSF have shown potential for distinguishing $\mathrm{AD}$ from controls $[139,140]$. Additionally, proteins with 3-NT adducts correlate with MMSE and thus show potential for disease staging or monitoring [140]. 


\section{F2-isoprostanes}

Other leading candidate markers in this area are protein-free. The most-studied are F2-isoprostanes, which are stable by-products of lipid peroxidation. CSF F2-isoprostanes are increased in $\mathrm{AD}$ [141], MCI/prodromal AD [142], and in presymptomatic individuals who later progress to $\mathrm{AD}$ [143] or who carry FAD mutations [144]. In addition to utility for diagnosis and prognosis, CSF F2-isoprostanes have recently shown promise as theragnostic markers of target engagement in a large randomized, double-blind, placebo-controlled clinical trial of a cocktail of antioxidants [145]. Although no positive impact on traditional/established AD biomarkers or on cognitive functioning was observed, levels of CSF F2-isoprostane levels did decrease in the treatment group.

\section{DNA \& RNA oxidation}

Somewhat less-studied as CSF biomarkers for oxidative damage in $\mathrm{AD}$ are by-products of DNA and RNA oxidation, 8-hydroxyguanine and 8-hydroxyguanosine (8-OHG). In studies of post-mortem brain tissue, levels of 8-OHG are increased in vulnerable neurons during the early stages of symptomatic AD (MCI) but not in preclinical stages [146]. To date, only one small study has reported data for CSF 8-OHG levels in AD. In this study, CSF 8-OHG was fivefold higher in AD compared with healthy controls, correlated with MMSE, and correlated inversely with subsequent disease duration (higher levels 'predicted' rapid progression) [147]. This candidate CSF marker likely lacks specificity for AD since it has also been reported to be elevated in Parkinson's disease [148], multiple-system atrophy [149] and in brain tissues affected by numerous neurodegenerative and inflammatory conditions [150]. However, as part of a panel of other markers that can confer disease specificity, 8-OHG might prove very useful for prognosis and theragnosis, if these results can be replicated.

\section{Novel candidate approaches}

Although the candidate markers discussed to this point remain incompletely vetted, they might nevertheless be considered somewhat conventional, insofar as they have been studied for some time and, by now, generally reflect hypothetical mechanisms associated with $\mathrm{AD}$ pathophysiology. By contrast, results from several newer approaches for CSF biomarker discovery have been reported recently, and some are worthy of discussion.

\section{Metabolome}

Continuing the 'omics' revolution that has been dominated by studies of DNA, RNA and protein, two studies have described changes in the metabolome of AD lumbar CSF. The metabolome refers to the set of small-molecule metabolites (such as metabolic intermediates or secondary metabolites) found within a biological sample in a particular physiologic or developmental state; thus, disease states that perturb biochemical networks will result in different metabolic signatures [151]. One study reported levels of eight amino acids (out of 32 examined) that were increased in $\mathrm{AD}$ versus $\mathrm{MCI}$ CSF (Table 1) [152]. Another much larger study (79 AD and 51 controls) measured 343 different analytes and also identified eight molecules with statistical significance (Table 1) [153]. As ensembles, subsets of these eight yielded sensitivities and specificities of $>80 \%$ for distinguishing $\mathrm{AD}$ from controls [153]. Additionally, one of these markers, cortisol, correlated with disease progression.

\section{miRNAs}

miRNAs have emerged as important indicators of physiologic and pathophysiologic stress [154]. miRNAs are small, noncoding RNA molecules that bind to their target mRNA and disrupt translation and stability [155]. Although miRNAs play important roles in the fine-tuning of normal gene expression, miRNA levels are often altered under disease conditions [156], and thus are increasingly being used as diagnostic and/or prognostic biomarkers in several fields. Various tissues, including brain, have been widely used for miRNA biomarker discovery. However, only a handful of studies have detected/reported miRNAs in CSF [157], mostly in assessing them as potential biomarkers of brain cancers [158-160]. To our knowledge, only a single published study has evaluated specific miRNAs in AD CSF [161]. In this study, 60 miRNAs were detected as being significantly different between control (nondemented, Braak stage 1) and AD (demented, Braak stage 5) samples, many of which are known to be involved in immune cell function. Although the correspondence between CSF miRNAs and underlying AD pathology remains unclear, future evaluations in well-characterized clinical cohorts will better define their potential as AD-related biomarkers, and perhaps provide insights into possible novel molecular systems as suitable targets for disease-modifying therapies. 


\section{Critical next steps}

The AD biomarker field is faced with several challenges it must overcome in order to move promising analytes into clinical practice. From a methodological perspective, biomarker candidates must be validated in large, well-characterized research cohorts, with care taken to evaluate the potential impact of $\mathrm{AD}$-related covariates such as age, gender and $A P O E$ genotype. Importantly, protocol and assay standardization must be achieved in order to maximize biomarker reliability and permit comparisons between studies. For example, since potential diurnal variations in CSF $A \beta$ levels have been reported [162] (but see [163]), sample collection and processing procedures must be standardized. In addition, significant between-assay and within-assay performance inconsistencies among different research laboratories must be overcome [164-167]. Quantitative assays with high sensitivity, precision and reliability must be developed and eventually transformed into platforms with high-throughput capabilities. Given the long preclinical stage of $\mathrm{AD}$, biomarker validation should define sensitivity and specificity (and associated positive and negative predictive values) for underlying $\mathrm{AD}$ pathology, not merely clinical diagnosis that is often inaccurate. Very few studies have been able to correlate biomarkers in ante-mortem samples with results of post-mortem examination, and the time interval between the two evaluations can be large. Thus, cross-sectional studies employing only clinical measures must be large to compensate for the loose correlation between functional and pathological decline. Methods to image neurofibrillary tangles and inflammatory responses in vivo are currently being developed and will be very informative for providing pathologic validation for CSF markers [168]. Evaluation of biomarker utility for differential diagnosis in cohorts with known underlying non-AD pathologies (FTLD, DLB, VaD, among others) is also needed. Characterizing the change in biomarker profiles with disease progression, both in terms of underlying pathology as well as severity of clinical symptoms, will be important for disease staging purposes, and large, longitudinal AD biomarker studies are currently underway in the USA and abroad, for example:

- Alzheimer's Disease Neuroimaging Initiative; (ADNI; USA, Europe, Japan);

- Adult Children Study (ACS);

- Alzheimer's Prevention Initiative (API);
- Arizona Alzheimer's Prevention Initiative APOE (Arizona API APOE);

- Australian Imaging Biomarkers and Lifestyle Study of Ageing (AIBL);

- Biomarkers for Older Controls at Risk for Dementia (BIOCARD);

- Development of Screening Guidelines and Criteria for Predementia Alzheimer's Disease (DESCRIPA);

- Dominantly Inherited Alzheimer Network (DIAN);

- Healthy Aging and Senile Dementia (HASD);

- Wisconsin Registry for Alzheimer Prevention (WRAP); and see [202].

Furthermore, change in analyte levels within individuals over time may be more useful as biomarkers than baseline measures alone [143,169-171].

It is highly likely that combinations of biomarkers will prove most useful for disease diagnosis (presence vs absence of AD pathology) and prognosis (predicting cognitive decline) $[24,29,78,116,172]$ within modalities or between modalities (e.g., fluids and imaging) [173-175]. Ongoing studies that collect multiple types of biomarker data will be critical for that assessment. To the extent that upcoming CSF biomarker candidates achieve significant diagnostic and prognostic value, it is likely that they will be considered in clinical trial design (for participant enrichment and target engagement) and evaluation of therapeutic efficacy as is currently being done with the more established analytes - A $\beta 42$, tau and $p$-tau.

\section{Future perspective}

For diagnosis and differential diagnosis of $\mathrm{AD}$, it is unlikely that CSF $A \beta 42$, tau and $p$-tau will be supplanted because they represent core features of AD pathology. More than likely, they will be complemented by markers that reflect the presence of more general neurodegenerative pathologies (e.g., neuroinflammation and synaptic loss/dysfunction) (FIGURE 2) in order to improve discrimination from healthy aging, and other markers with some specificity for other causes of dementia (e.g., Lewy body diseases, TDP-43-opathies, other tauopathies and vascular disease). Specific markers for non-AD dementias have received somewhat less research attention in the past due to limited CSF collections, but several efforts focused on DLB and FTLD are underway and will facilitate such biomarker discovery. Identifying biomarker panels that can diagnose mixed dementias will likely 
prove somewhat more difficult, but the task is not intractable.

The hunt for prognostic markers, like that for non-AD dementias, has been slowed by a limited number of CSF samples from participants with sufficient longitudinal clinical follow-up. Fortunately, this problem is being solved by patience. Even over the last 5 years, several large collections have matured, and the technology for novel biomarker discovery has advanced sufficiently to allow several candidate prognostic markers to be identified.

As discussed above, several novel markers with potential for staging cohorts have been identified [75,77,95], and a robust panel of candidates, validated in multiple cohorts, may be on the 5-year horizon. Markers for monitoring longitudinal progression within individuals, from cognitive normalcy through mild dementia, will take longer; the cohorts required for such studies necessarily require the passage of time as pathology evolves. Some biorepositories presently hold a limited number of such samples, but more will be required for statistical rigor and for validation in independent cohorts.

Finally, with the advancement of several potential disease-modifying therapies into Phase II and III, CSF samples from patients enrolled in clinical

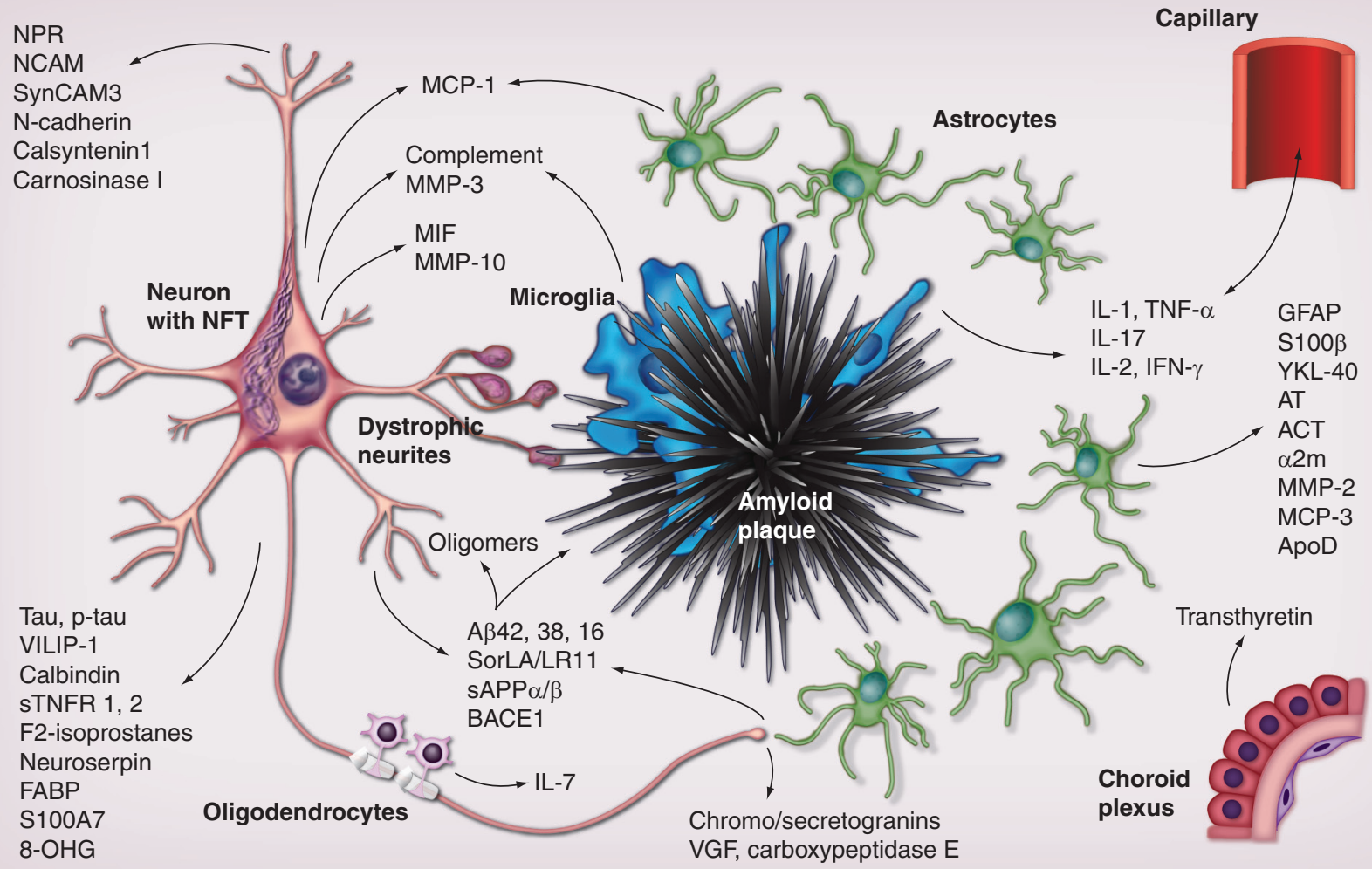

Biomarkers Med. (c) Future Science Group (2012)

Figure 2. Histological representation of origins of upcoming and established cerebrospinal fluid biomarkers. Synaptic adhesion molecules, other neuronal proteins, and dense core vesicle proteins generally decline in $A D$, perhaps reflecting synapse loss (upper left and bottom center, respectively). Other neuron-derived proteins (bottom left), including tau and p-tau, and products of oxidative damage, increase in AD and may reflect neuronal injury. Also produced by neurons, A 342 (center left) preferentially oligomerizes and partitions into plaques, but other candidate biomarker proteins associated with APP processing, including shorter A $\beta$ species, SorLA, soluble fragments of APP and secreted BACE1, do not. Different subsets of neuroinflammatory molecules are secreted by each of the parenchymal cells represented here; neurons selectively produce MIF and MMP-10 (upper center), but share responsibility with astrocytes for MCP-1 and with microglial cells for MMP-3 and factors of complement. Microglial cells themselves produce most of the other cytokines and chemokines (upper right), though many of these can also cross the blood-brain barrier from the periphery. Among the neuroinflammatory mediators of greater significance as potential biomarkers, oligodendroglial cells produce IL-7 (lower left), and astrocytes produce MMP-2, MMP-3 and YKL-40 (center right). Astrocytes are also responsible for shedding some intrinsic proteins (GFAP, S100 $\beta$ ) and for secreting many other miscellaneous proteins implicated in AD pathogenesis. Transthyretin (lower right), one of the most abundant proteins in the CSF, is produced principally by the choroid plexus. Not pictured are many other candidate biomarker molecules for which the cell(s) of origin are unknown (e.g., miRNAs), not specific (e.g., cystatin C) or too prolific (e.g., astrocyte products), prohibiting the inclusion of all their relevant secreted products.

8-OHG: 8-hydroxyguanosine; A $\beta$ : $\beta$-amyloid; ACT: Antichymotrypsin; AT: Antitrypsin; NFT: Neurofibrillary tangles; p-tau: Phosphorylated tau; sTNFR: Soluble TNF- $\alpha$ receptor. 


\section{Executive summary}

\section{The crisis of Alzheimer's disease}

- Alzheimer's disease (AD) dementia will soon become a public health crisis if disease-modifying treatments are not developed.

- Methods are needed to increase limited clinical diagnostic accuracy to improve patient care and aid in therapeutic clinical trial design.

- AD clinical trials to date have been largely unsuccessful, likely because enrolled participants already exhibit mild/moderate dementia and thus significant neuron death.

\section{$A D$ is a chronic disease}

- AD dementia is the end stage of a long, chronic disease process.

- AD has a long asymptomatic ('preclinical') stage during which $\beta$-amyloid and tangle pathologies develop, prior to significant neuron death and cognitive symptoms.

- Preclinical diagnosis will afford emerging disease-modifying therapies the best chance of preserving normal cognitive function.

\section{Established cerebrospinal fluid biomarkers of $A D$}

- A biomarker is a characteristic that is objectively measured and evaluated as an indicator of normal biological processes, pathogenic processes or pharmacologic responses to a therapeutic intervention.

- Biomarkers can be used to guide clinical diagnosis (diagnostic), estimate disease risk or prognosis (prognostic), evaluate disease stage and monitor progression and/or response to therapy (theragnostic).

- The most established AD biomarkers are cerebrospinal fluid (CSF) levels of $\beta$-amyloid 42 (A $\beta 42$ ), total tau and phosphorylated tau ( $p$-tau).

- $A D$ is characterized by low levels of CSF A $\beta 42$ (a marker of $\beta$-amyloid plaques) and high levels of tau(s) (markers of neurofibrillary tangles and/or neurodegeneration).

- The CSF tau:A 342 ratio is predictive of cognitive decline in cognitively normal elders and progression to AD dementia in individuals with mild cognitive impairment.

\section{The need for novel biomarkers}

- Reported biomarker sensitivities and specificities for AD clinical diagnosis vary widely.

- Novel biomarkers are needed to identify additional pathogenic processes that will increase diagnostic/prognostic accuracy, aid in differential diagnosis, identify cases with mixed pathologies and define the trajectory of biomarker changes over time.

\section{Upcoming candidate CSF biomarkers}

- Beyond CSF A 342 , tau and p-tau181, many analytes have shown promise and are worthy of future study.

- Several promising CSF biomarkers are related to APP processing, A $\beta$ metabolism and/or amyloidogenesis.

- CSF levels of certain phosphorylated forms of tau may perform better than others in identifying tangle pathology and for differential diagnosis.

- Many promising analytes are putative markers of synapse loss and/or neurodegeneration.

- Several apolipoproteins in CSF have shown AD-associated changes.

- Many neuroinflammatory molecules have been identified as potential CSF biomarkers.

- Potential markers of pathological oxidative processes have also been identified.

- Novel biomarker approaches of considerable interest but in need of further method development include 'metabolome' screens and surveys of miRNAs.

\section{Critical next steps}

- Protocol and assay standardization must be achieved.

- Biomarker candidates must be validated in large, well-characterized research cohorts, taking into consideration important covariates such as age, gender and $A P O E$ genotype.

- Biomarker validation must define sensitivity and specificity for underlying pathologies, not just clinical diagnosis, which is often inaccurate.

- Biomarkers that correlate with cognitive measures are needed to aid in dementia staging.

- Important next steps include evaluation of biomarker change over time within individuals and investigation of the utility of biomarker combinations for AD diagnosis/prognosis.

- Once validated, markers should be considered for use in clinical trial design and therapeutic evaluation.

\section{Future perspective}

- The combination of CSF A 42 and tau will remain the essential core AD biomarkers.

- The addition of novel biomarkers will likely increase diagnostic/prognostic accuracy by identifying non-AD pathologies.

- Evaluation of biomarker panels for diagnosing mixed dementias will likely be in progress.

- Longitudinal biomarker studies will mature and permit discovery of prognostic panels that will be useful for disease staging, especially in very early and preclinical stages.

- CSF obtained in clinical trials will be evaluated for proof of target engagement and evidence of therapeutic effects on downstream pathologic processes.

- Once effective disease-modifying therapies have been developed, validated CSF biomarkers with high sensitivity and specificity for AD will likely be utilized in the clinical setting to aid disease diagnosis and prognosis, as has recently been proposed. 
trials will be interrogated for various biomarkers (theragnostic) as proof of target engagement and evidence of therapeutic effects on downstream pathobiologic processes $[145,176]$. Since biomarkers are likely to be applied as panels to maximize accuracy, robust panels that emerge for clinical trials or patient care will reflect a combination of empirical and idiosyncratic complementarity. More practical issues, such as molecular stability, simplicity of measurement, accessibility and reproducibility of measurement platform, readiness for implementation when clinical trials are planned and executed, marketing and cost must also be considered. Resolution of these issues must involve a coordinated effort among academic research laboratories, pharma and commercial assay developers, as is currently being pursued for the more established CSF biomarkers [167]. To the extent that these goals can be met, CSF biomarkers, be they 'established' or 'upcoming', may soon be applied in clinical settings, as has recently been proposed [177].

\section{Financial \& competing interests disclosure}

AM Fagan is supported by grants from the National Institute of Aging of the National Institutes of Health (PO1 AG03991, P01 AG026276 and U01 AG032438) and the Hope Center for Neurological Disorders, and is a member of the Alzheimer's Disease CSF Biomarker Development Advisory Board for Roche and the US Alzheimer's Disease Advisory Board for Lilly USA. No conflict of interest exists. RJ Perrin is supported by a grant from the National Institute of Aging of the National Institutes of Health (P50 AG05681). He reports no conflicts of interest. The authors have no other relevant affiliations or financial involvement with any organization or entity with a financial interest in or financial conflict with the subject matter or materials discussed in the manuscript apart from those disclosed.

No writing assistance was utilized in the production of this manuscript.

\section{References}

Papers of special note have been highlighted as: - of interest

" " of considerable interest

1 Brookmeyer R, Evans D, Hebert L et al. National estimates of the prevalence of Alzheimer's disease in the United States. Alzheimers Dement. 1, 61-73 (2011).

2 McKhann G, Drachman D, Folstein M, Katzman R, Price D, Stadlan E. Clinical diagnosis of Alzheimer's disease: Report of the NINCDS-ADRDA Work Group under the auspices of Department of Health and Human Services Task Force on Alzheimer's Disease. Neurology 34, 939-944 (1984).

3 Beach T, Monsell S, Phillips L, Kukull W. Accuracy of the clinical diagnosis of Alzheimer disease at National Institute on Aging Alzheimer Disease Centers, 2005-2010. J. Neuropathol. Exp. Neurol. 71, 266-273 (2012).

4 Price J, Ko A, Wade M, Tsou S, McKeel D, Morris J. Neuron number in the entorhinal cortex and CA1 in preclinical Alzheimer's disease. Arch. Neurol. 58, 1395-1402 (2001).

5 Petersen R, Smith G, Waring S, Ivnik R, Tabgalos E, Kokmen E. Mild cognitive impairment: clinical characterization and outcome. Arch. Neurol. 56, 303-308 (1999).

6 Price J, Morris J. Tangles and plaques in nondemented aging and 'preclinical' Alzheimer's disease. Ann. Neurol. 45, 358-368 (1999).

7 Hulette C, Welsh-Bohmer K, Murray M, Saunders A, Mash D, McIntyre L.
Neuropathological and neuropsychological changes in 'normal' aging: evidence for preclinical Alzheimer disease in cognitively normal individuals. J. Neuropathol. Exp. Neurol. 57, 1168-1174 (1998).

8 Gomez-Isla T, Price J, McKeel D, Morris J, Growdon J, Hyman B. Profound loss of layer II entorhinal cortex neurons occurs in very mild Alzheimer's disease. J. Neurosci. 16, 4491-4500 (1996).

9 Braak H, Braak E. Frequency of stages of Alzheimer-related lesions in different age categories. Neurobiol. Aging 18, 351-357 (1997).

10 Markesbery W, Schmitt F, Kryscio R, Davis D Smith C, Wekstein D. Neuropathologic substrate of mild cognitive impairment. Arch. Neurol. 63, 38-46 (2006).

11 Biomarkers Definitions Working Group. Biomarkers and surrogate endpoints: preferred definitions and conceptual framework. Clin. Pharmacol. Ther. 69, 89-95 (2001).

12 Blennow K, Hampel H, Weiner M, Zetterberg $H$. Cerebrospinal fluid and plasma biomarkers for Alzheimer's disease. Nat. Rev. Neurol. 6, 131-144 (2010).

13 Sunderland T, Linker G, Mirza N et al. Decreased $\beta$-amyloid1-42 and increased tau levels in cerebrospinal fluid of patients with Alzheimer's disease. JAMA 289, 2094-2103 (2003).

14 Clark C, Xie S, Chittams J et al. Cerebrospinal fluid tau and beta-amyloid: how well do these biomarkers reflect autopsy-confirmed dementia diagnoses? Arch. Neurol. 60, 1696-1702 (2003).
15 Fagan A, Mintun M, Mach R et al. Inverse relation between in vivo amyloid imaging load and CSF A 442 in humans. Ann. Neurol. 59, 512-519 (2006).

16 Forsberg A, Engler H, Almkvist O et al. PET imaging of amyloid deposition in patients with mild cognitive impairment. Neurobiol. Aging 29, 1456-1465 (2008).

17 Tolboom N, Vander Flier W, Yaqub M et al. Relationship of cerebrospinal fluid markers to ${ }^{11} \mathrm{C}-\mathrm{PiB}$ and ${ }^{18} \mathrm{~F}-\mathrm{FDDNP}$ binding. J. Nucl. Med. 50, 1464-1470 (2009).

18 Grimmer T, Riemenschneider M, Förstl H et al. Beta amyloid in Alzheimer's disease: increased deposition in brain is reflected in reduced concentration in cerebrospinal fluid. Biol. Psychiatry 65, 927-934 (2009).

19 Jagust W, Landau S, Shaw L et al. Relationships between biomarkers in aging and dementia. Neurology 73, 1193-1199 (2009).

20 Hesse C, Rosengren L, Vanmechelen E et al. Cerebrospinal fluid markers for Alzheimer's disease evaluated after acute ischemic stroke. J. Alzheimers Dis. 2, 199-206 (2000).

21 Ost M, Nylén K, Csajbok L et al. Initial CSF total tau correlates with 1-year outcome in patients with traumatic brain injury. Neurology 67, 1600-1604 (2006).

22 Buerger K, Ewers M, Pirttila T et al. CSF phosphorylated tau protein correlates with neocortical neurofibrillary pathology in Alzheimer's disease. Brain 129, 3035-3041 (2006).

23 Blom E, Giedraitis V, Zetterberg $\mathrm{H}$ et al. Rapid progression from mild cognitive impairment to Alzheimer's disease in subjects 
with elevated levels of tau in cerebrospinal fluid and the APOE epsilon4/epsilon4 genotype. Dement. Geriatr. Cogn. Disord. 27, 458-464 (2009).

24 Hansson O, Zetterberg H, Buchhave P, Londos E, Blennow K, Minthon L. Association between CSF biomarkers and incipient Alzheimer's disease in patients with mild cognitive impairment: a follow-up study. Lancet Neurol. 5, 228-234 (2006).

25 Fagan A, Roe C, Xiong C, Mintun M, Morris J, Holtzman D. Cerebrospinal fluid tau/A $\beta 42$ ratio as a prediction of cognitive decline in nondemented older adults. Arch. Neurol. 64, 343-349 (2007).

26 Li G, Sokal I, Quinn J et al. CSF tau/A $\beta 42$ ratio for increased risk of mild cognitive impairment: a follow-up study. Neurology 69, 631-639 (2007).

27 Craig-Schapiro R, Perrin R, Roe C et al. YKL-40: a novel prognostic fluid biomarker for preclinical Alzheimer's disease. Biol. Psychiatry 68, 903-912 (2010).

28 Snider B, Fagan A, Roe C et al. Cerebrospinal fluid biomarkers and rate of cognitive decline in very mild dementia of the Alzheimer type. Arch. Neurol. 66, 638-645 (2009).

29 Tarawneh R, D’Angelo G, Macy E et al. Visinin-like protein 1: a novel prognostic biomarker in Alzheimer's disease. Ann. Neurol. 70, 274-285 (2011).

- VILIP-1, comparable to tau, is one of the most promising upcoming novel candidates for Alzheimer's disease (AD) diagnosis, differential diagnosis and prognosis.

30 Landau S, Harvey D, Madison C et al. Comparing predictors of conversion and decline in mild cognitive impairment. Neurology 75, 230-238 (2010).

31 Buchhave P, Minthon L, Zetterberg H, Wallin A, Blennow K, Hansson O. Cerebrospinal fluid levels of $\beta$-amyloid 1-42, but not of Tau, are fully changed already 5 to 10 years before the onset of Alzheimer dementia. Arch. Gen. Psychiatry 69, 98-106 (2012).

32 Rossum IV, Vos S, Handels R, Visser P. Biomarkers as predictors for conversion from mild cognitive impairment to Alzheimer-type dementia: implications for trial design. J. Alzheimers Dis. 20, 881-891 (2010).

33 O'Brien R, Wong P. Amyloid precursor protein processing and Alzheimer's disease. Annu. Rev. Neurosci. 34, 185-204 (2011).

34 Lewczuk P, Kamrowski-Kruck H, Peters O et al. Soluble amyloid precursor proteins in the cerebrospinal fluid as novel potential biomarkers of Alzheimer's disease: a multicenter study. Mol. Psychiatry 15, 138-145 (2010).
35 Rosén C, Andreasson U, Mattsson $\mathrm{N}$ et al. Cerebrospinal fluid profiles of amyloid $\beta$-related biomarkers in Alzheimer's disease. Neuromol. Med. 14, 65-73 (2012).

36 Gabelle A, Roche S, Gény C et al. Correlations between soluble $\alpha / \beta$ forms of amyloid precursor protein and $A \beta 38,40$, and 42 in human cerebrospinal fluid. Brain Res. 1357, 175-183 (2010).

37 Zetterberg $\mathrm{H}$, Andreasson U, Hansson O et al. Elevated cerebrospinal fluid BACE1 activity in incipient Alzheimer disease. Arch. Neurol. 65, 1102-1107 (2008).

38 Olsson A, Höglund K, Sjögren M et al. Measurement of alpha- and beta-secretase cleaved amyloid precursor protein in cerebrospinal fluid from Alzheimer patients. Exp. Neurol. 183(74-80), (2003).

39 Perneczky R, Tsolakidou A, Arnold A et al. CSF soluble amyloid precursor proteins in the diagnosis of incipient Alzheimer disease. Neurology 77, 35-38 (2011).

40 Alexopoulos P, Guo L-H, Tsolakidou A et al. Interrelations between CSF soluble $\mathrm{A} \beta \mathrm{PP} \beta$, amyloid- $\beta$ 1-42, SORL1, and tau levels in Alzheimer's disease. J. Alzheimers Dis. 28, 543-552 (2011).

41 Vassar R, Kovacs D, Yan R, Wong P. The beta-secretase enzyme BACE in health and Alzheimer's disease: regulation, cell biology, function, and therapeutic potential. J. Neurosci. 29, 12787-12794 (2009).

42 Holsinger R, McLean C, Collins S, Masters C, Evin G. Increased beta-secretase activity in cerebrospinal fluid of Alzheimer's disease subjects. Ann. Neurol. 55, 898-899 (2004).

43 Holsinger R, Lee J, Boyd A, Masters C, Collins S. CSF BACE1 activity is increased in CJD and Alzheimer disease versus other dementias. Neurology 67, 710-712 (2006).

44 Verheijen J, Huisman L, Lent NV et al. Detection of a soluble form of BACE-1 in human cerebrospinal fluid by a sensitive activity assay. Clin. Chem. 6, 1168-1174 (2006).

45 Zhong Z, Ewers M, Teipel S et al. Levels of beta-secretase (BACE1) in cerebrospinal fluid as a predictor of risk in mild cognitive impairment. Arch. Gen. Psychiatry 64, 718-726 (2007).

46 Mulder S, van der Flier W, Verheijen J et al. BACE1 activity in cerebrospinal fluid and its relation to markers of AD pathology. J. Alzheimers Dis. 1, 253-260 (2010).

47 Qin W, Ho L, Wang J, Peskind E, Pasinetti G. S100A7, a novel Alzheimer's disease biomarker with non-amyloidogenic alpha-secretase activity acts via selective promotion of ADAM-10. PLoS One 4, e4183 (2009).
48 Watson P, Leygue E, Murphy L. Psoriasin (S100A7). Int. J. Biochem. Cell Biol. 30, 567-571 (1998).

49 Schmidt V, Sporbert A, Rohe M et al. SorLA/ LR11 regulates processing of amyloid precursor protein via interaction with adaptors GGA and PACS-1. J. Biol. Chem. 45, 32956-32964 (2007).

50 Dodson S, Gearing M, Lippa C, Montine T, Levey A, Lah J. LR11/SorLA expression is reduced in sporadic Alzheimer disease but not in familial Alzheimer disease. J. Neuropathol. Exp. Neurol. 9, 866-872 (2006).

51 Scherzer C, Offe K, Gearing M et al. Loss of apolipoprotein E receptor LR11 in Alzheimer disease. Arch. Neurol. 61, 1200-1205 (2004).

52 Rogaeva E, Meng Y, Lee J et al. The neuronal sortilin-related receptor SORL1 is genetically associated with Alzheimer disease. Nat. Genet. 39, 168-177 (2007).

53 Ma Q, Galasko D, Ringman J et al. Reduction of SorLA/LR11, a sorting protein limiting beta-amyloid production, in Alzheimer disease cerebrospinal fluid. Arch. Neurol. 4, 448-457 (2009).

54 Ikeuchi T, Hirayama S, Miida T et al. Increased levels of soluble LR11 in cerebrospinal fluid of patients with Alzheimer disease. Dement. Geriatr. Cogn. Disord. 1, 28-32 (2010).

55 Fagan A, Mintun M, Shah A et al. Cerebrospinal fluid tau and ptau181 increase with cortical amyloid deposition in cognitively normal individuals: Implications for future clinical trials of Alzheimer's disease. EMBO Mol. Med. 1, 371-380 (2009).

56 Lewczuk P, Esselmann H, Meyer M et al. The amyloid- $\beta(A \beta)$ peptide pattern in cerebrospinal fluid in Alzheimer's disease: evidence of a novel carboxyterminally elongated A $\beta$ peptide. Rapid Commun. Mass Spectrom. 17, 1291-1296 (2003).

57 Schoonenboom N, Mulder C, Kamp GV et al. Amyloid $\beta$ 38, 40, and 42 species in cerebrospinal fluid: more of the same? Ann. Neurol. 58, 139-142 (2005).

58 Portelius E, Westman-Brinkmalm A, Zetterberg H, Blennow K. Determination of $\beta$-amyloid peptide signatures in cerebrospinal fluid using immunoprecipitation-mass spectrometry. J. Proteome Res. 5, 1010-1016 (2006).

59 Portelius E, Zetterberg $\mathrm{H}$, Andreasson U et al. An Alzheimer's disease-specific beta-amyloid fragment signature in cerebrospinal fluid. Neurosci. Lett. 409, 215-219 (2006).

60 Portelius E, Andreasson U, Ringman J et al. Distinct cerebrospinal fluid amyloid beta peptide signatures in sporadic and PSEN1 A431E-associated familial Alzheimer's disease. Mol. Neurodegener. 5, 2 (2010). 
61 Portelius E, Zetterberg H, Dean R et al. Amyloid- $\beta 1-15 / 16$ as a marker for $\gamma$-secretase inhibition in Alzheimer's disease. J. Alzheimers Dis. doi:10.3233/JAD-2012-120508 (2012) (Epub ahead of print).

- Demonstrates cerebrospinal fluid (CSF) A $\beta 1-15 / 16$ as a potential theragnostic marker in individuals treated with a $\gamma$-secreatase inhibitior.

62 Walsh D, Selkoe D. A beta oligomers - a decade of discovery. J. Neurochem. 101, 1172-1184 (2007).

63 Benilova I, Karran E, De Strooper B. The toxic $\mathrm{A} \beta$ oligomer and Alzheimer's disease: an emperor in need of clothes. Nat. Neurosci. 15, 349-357 (2012).

64 Georganopoulou D, Chang L, Nam J et al. Nanoparticle-based detection of a solube pathogenic biomarker for Alzheimer's disease. Proc. Natl Acad. Sci. USA 102, 2273-2276 (2005).

65 Klyubin I, Betts V, Welzel A et al. Amyloid beta protein dimer-containing human CSF disrupts synaptic plasticity: prevention by systemic passive immunization. J. Neurosci. 28, 4231-4237 (2008).

66 Fukumoto H, Tokuda T, Kasai T et al. High-molecular-weight beta-amyloid oligomers are elevated in cerebrospinal fluid of Alzheimer patients. FASEB J. 24, 2716-2726 (2010).

67 Santos A, Ewers M, Minthon L et al. Amyloid- $\beta$ oligomers in cerebrospinal fluid are associated with cognitive decline in patients with Alzheimer's disease. J. Alzheimers Dis. 1, 171-176 (2012).

68 Gao C, Yam A, Wang X et al. A 340 oligomers identified as a potential biomarker for the diagnosis of Alzheimer's disease. PLoS One 5 , e15725 (2010).

69 Kinghorn K, Crowther D, Sharp L et al. Neuroserpin binds Abeta and is a neuroprotective component of amyloid plaques in Alzheimer disease. J. Biol. Chem. 39, 2926829277 (2006).

70 Fabbro S, Seeds N. Plasminogen activator activity is inhibited while neuroserpin is up-regulated in the Alzheimer disease brain. J. Neurochem. 2, 303-315 (2009).

71 Nielsen H, Minthon L, Londos E et al. Plasma and CSF serpins in Alzheimer disease and dementia with Lewy bodies. Neurology 16, 1569-1579 (2007).

72 Finehout EJ, Franck Z, Choe LH, Relkin N, Lee KH. Cerebrospinal fluid proteomic biomarkers for Alzheimer's disease. Ann. Neurol. 61, 120-129 (2007).

73 Zhang J, Goodlett D, Quinn J et al. Quantitative proteomics of cerebrospinal fluid from patients with Alzheimer's disease. J. Alzheimers Dis. 7, 125-133 (2005).
74 Puchades M, Hansson S, Nilsson C, Andreasen N, Blennow K, Davidsson P. Proteomic studies of potential cerebrospinal fluid protein markers for Alzheimer's disease. Mol. Brain Res. 118, 140-146 (2003).

75 Perrin R, Craig-Schapiro R, Malone J et al. Identification and validation of novel cerebrospinal fluid biomarkers for staging early Alzheimer's disease. PLoS One 6, e16032 (2011).

-1 Large 2D-DIGE study identifies 47 candidate markers for AD versus control; ELISA validation in independent cohort identifies biomarker patterns associated with six clinical stages of $\mathrm{AD}$.

76 Hansson S, Andréasson U, Wall M et al. Reduced levels of amyloid-beta-binding proteins in cerebrospinal fluid from Alzheimer's disease patients. J. Alzheimers Dis. 2, 389-397 (2009).

$77 \mathrm{Hu}$ W, Chen-Plotkin A, Arnold S et al. Novel CSF biomarkers for Alzheimer's disease and mild cognitive impairment. Acta Neuropathol. 119, 669-678 (2010).

"- Evaluates $>150$ autopsy-verified ante-mortem $\mathrm{AD}$, non- $\mathrm{AD}$ and control samples for 151 analytes in a CLIA-approved setting; identifies candidate markers and biomarker panels for diagnosis, differential diagnosis, staging and prognosis.

78 Craig-Schapiro R, Kuhn M, Xiong C et al. Multiplexed immunoassay panel identifies novel CSF biomarkers for Alzheimer's disease diagnosis and prognosis. PLoS One 6, e18850 (2011).

-" Evaluates 333 well-characterized AD and control samples for 193 analytes in a clinical laboratory improvement amendmentsapproved setting; identifies candidate markers and biomarker panels that improve upon tau and $\mathrm{A} \beta 42$ for diagnosis and prognosis.

$79 \mathrm{Hu}$ Y, Malone J, Fagan A, Townsend R, Holtzman D. Comparative proteomic analysis of intra- and interindividual variation in human cerebrospinal fluid. Mol. Cell. Proteom. 4, 2000-2009 (2005).

80 Simonsen A, McGuire J, Hansson O et al. Novel panel of cerebrospinal fluid biomarkers for the prediction of progression to Alzheimer dementia in patients with mild cognitive impairment. Arch. Neurol. 64, 366-370 (2007).

81 Simonsen A, McGuire J, Podust V et al. Identification of a novel panel of cerebrospinal fluid biomarkers for Alzheimer's disease. Neurobiol. Aging 29, 961-968 (2008).

82 Carrette O, Demalte I, Scherl A et al. A panel of cerebrospinal fluid potential biomarkers for the diagnosis of Alzheimer's disease. Proteomics 3, 1486-1494 (2003).
83 Abdi F, Quinn J, Jankovic J et al. Detection of biomarkers with a multiplex quantitative proteomic platform in cerebrospinal fluid of patients with neurodegenerative disorders. J. Alzheimers Dis. 9, 293-348 (2006).

- Compares 1500 proteins among AD, dementia with Lewy bodies, Parkinson's disease and controls; reports many individual markers and small, highly accurate biomarker panels for differential diagnosis.

84 Davidsson P, Westman-Brinkmalm A, Nilsson C et al. Proteome analysis of cerebrospinal fluid proteins in Alzheimer patients. NeuroReport 13, 611-615 (2002).

85 Hu Y, Hosseini A, Kauwe J et al. Identification and validation of novel CSF biomarkers for early stages of Alzheimer's disease. Proteomics Clin. Appl. 1, 1373-1384 (2007).

86 Ji L, Chauhan A, Wegiel J, Essa M, Chauhan V. Gelsolin is proteolytically cleaved in the brains of individuals with Alzheimer's disease. J. Alzheimers Dis. 18, 105-111 (2009).

87 Buerger K, Alafuzoff I, Ewers M, Pirttilä T, Zinkowski R, Hampel H. No correlation between CSF tau protein phosphorylated at threonine 181 with neocortical neurofibrillary pathology in Alzheimer's disease. Brain 130, e82 (2007).

88 Hampel H, Buerger K, Zinkowski R et al. Measurement of phosphorylated tau epitopes in the differential diagnosis of Alzheimer disease: a comparative cerebrospinal fluid study. Arch. Gen. Psychiatry 1, 95-102 (2004).

89 Buerger K, Zinowski R, Teipel S et al. Differential diagnosis of Alzheimer's disease with cerebrospinal fluid levels of tau protein phosphorylated at threonine 231. Arch. Neurol. 59, 1267-1272 (2002).

90 Buerger K, Zinkowski R, Teipel S et al. Differentiation of geriatric major depression from Alzheimer's disease with CSF tau protein phosphorylated at threonine 231 . Am. J. Psychiatry 160, 376-379 (2003).

91 De Leon MJ, Segal S, Tarashish CY et al. Longitudinal cerebrospinal fluid tau load increases in mild cognitive impairment. Neurosci. Lett. 333, 183-186 (2002).

92 Buerger K, Teipel S, Zinkowski R et al. CSF tau protein phosphorylated at threonine 231 correlates with cognitive decline in MCI subjects. Neurology 59, 627-629 (2002).

93 Ewers M, Buerger K, Teipel S et al. Multicenter assessment of CSFphosphorylated tau for the prediction of conversion of MCI. Neurology 24, 2205-2212 (2007).

94 Kovacech B, Novak M. Tau truncation is a productive posttranslational modification of 
neurofibrillary degeneration in Alzheimer's disease. Curr. Alzheimer Res. 8, 708-716 (2010).

$95 \mathrm{Hu} \mathrm{W}$, Chen-Plotkin A, Arnold S et al. Biomarker discovery for Alzheimer's disease, frontotemporal lobar degeneration, and Parkinson's disease. Acta Neuropathol. 120, 385-399 (2010).

96 Jahn H, Wittke S, Zürbig P et al. Peptide fingerprinting of Alzheimer's disease in cerebrospinal fluid: identification and prospective evaluation of new synaptic biomarkers. PLoS One 10, e26540 (2011).

97 Yin G, Lee H, Cho J, Suk K. Neuronal pentraxin receptor in cerebrospinal fluid as a potential biomarker for neurodegenerative diseases. Brain Res. 1265, 158-170 (2009).

98 Thorsell A, Bjerke M, Gobom J et al. Neurogranin in cerebrospinal fluid as a marker of synaptic degeneration in Alzheimer's disease. Brain Res. 1362, 13-22 (2010).

99 Tarawneh R, Lee J, Ladenson J, Morris J, Holtzman D. CSF VILIP-1 predicts rates of cognitive decline in early Alzheimer disease. Neurology 10, 709-719 (2012).

100 Kim J, Basak J, Holtzman D. The role of apolipoprotein E in Alzheimer's disease. Neuron 63, 287-303 (2009).

101 Wu Z, Yu J, Li Y, Tan L. Clusterin in Alzheimer's disease. Adv. Clin. Chem. 56, 155-173 (2012).

102 Castellano J, Kim J, Stewart F et al. Human apoE isoforms differentially regulate brain amyloid- $\beta$ peptide clearance. Sci. Transl. Med. 3, 89ra57 (2011).

103 Terrisse L, Poirier J, Bertrand P et al. Increased levels of apolipoprotein $\mathrm{D}$ in cerebrospinal fluid and hippocampus of Alzheimer's patients. J. Neurochem. 71, 1643-1650 (1998).

104 Wang Y, Hancock A, Bradner J et al. Complement 3 and factor $h$ in human cerebrospinal fluid in Parkinson's disease, Alzheimer's disease, and multiple-system atrophy. Am. J. Pathol. 4, 1509-1516 (2011).

105 Licastro F, Parnetti L, Morini M et al. Acute phase reactant alpha 1-antichymotrypsin is increased in cerebrospinal fluid and serum of patients with probable Alzheimer disease. Alzheimer Dis. Assoc. Disord. 9, 112-118 (1995).

106 Harigaya Y, Shoji M, Nakamura T, Matsubara E, Hosoda K, Hirai S. Alpha 1 -antichymotrypsin level in cerebrospinal fluid is closely associated with late onset Alzheimer's disease. Intern. Med. 34, 481-484 (1995).

107 Dekosky S, Ikonomovic M, Wang X et al. Plasma and cerebrospinal fluid $\alpha 1$ - antichymotrypsin levels in Alzheimer's disease: correlation with cognitive impairment. Ann. Neurol. 53, 81-90 (2003).

108 Mlekusch R, Humpel C. Matrix metalloproteinases- 2 and -3 are reduced in cerebrospinal fluid with low betaamyloid1-42 levels. Neurosci. Lett. 466, 135-138 (2009).

109 Swardfager W, Lanctôt K, Rothenburg L, Wong A, Cappell J, Herrmann N. A meta-analysis of cytokines in Alzheimer's disease. Biol. Psychiatry 68, 930-941 (2010).

110 Galasko D, Montine T. Biomarkers of oxidative damage and inflammation in Alzheimer's disease. Future Med. 4, 27-36 (2010).

111 Olson L, Humpel C. Growth factors and cytokines/chemokines as surrogate biomarkers in cerebrospinal fluid and blood for diagnosing Alzheimer's disease and mild cognitive impairment. Exp. Gerontol. 1, 41-46 (2010).

112 Popp J, Bacher M, Kölsch H et al. Macrophage migration inhibitory factor in mild cognitive impairment and Alzheimer's disease. J. Psychiatr. Res. 8, 749-753 (2009).

113 Tan Z, Beiser A, Vasan R et al. Inflammatory markers and the risk of Alzheimer disease: the Framingham Study. Neurology 68, 1902-1908 (2007).

114 Llano D, Li J, Waring J et al. Cerebrospinal fluid cytokine dynamics differ between Alzheimer disease patients and elderly controls. Alzheimer Dis. Assoc. Disord. doi:10.1097/WAD.0b013e31823b2728 (2011) (Epub ahead of print).

115 Bateman R, Siemers E, Mawuenyega K et al. A gamma-secretase inhibitor decreases amyloid-beta production in the central nervous system. Ann. Neurol. 66, 48-54 (2009).

116 Westin K, Buchhave P, Nielsen H, Minthon $\mathrm{L}$, Janciauskiene S, Hansson O. CCL2 is associated with a faster rate of cognitive decline during early stages of Alzheimer's disease. PLoS One 1, e30525 (2012).

$117 \mathrm{Hu}$ W, Chen-Plotkin A, Grossman M et al. Novel CSF biomarkers for frontotemporal lobar degenerations. Neurology 23, 2079-2086 (2010).

118 Filipovic R, Jakovcevski I, Zecevic N. GRO-alpha and CXCR2 in the human fetal brain and multiple sclerosis lesions. Dev. Neurosci. 25, 279-290 (2003).

119 Jiang H, Hampel H, Prvulovic D et al. Elevated CSF levels of TACE activity and soluble TNF receptors in subjects with mild cognitive impairment and patients with Alzheimer's disease. Mol. Neurodegener. 6, 69 (2011).
120 Buchhave P, Zetterberg H, Blennow K, Minthon L, Janciauskiene S, Hansson O. Soluble TNF receptors are associated with $\mathrm{A} \beta$ metabolism and conversion to dementia in subjects with mild cognitive impairment. Neurobiol. Aging 31, 1877-1884 (2010).

121 van Eijk J, van Everbroeck B, Abdo W, Kremer B, Verbeek M. CSF neurofilament proteins levels are elevated in sporadic Creutzfeldt-Jakob disease. J. Alzheimers Dis. 21, 569-576 (2010).

122 Jesse S, Steinacker P, Cepek L et al. Glial fibrillary acidic protein and protein S-100B: different concentration pattern of glial proteins in cerebrospinal fluid of patients with Alzheimer's disease and Creutzfeldt-Jakob disease. J. Alzheimers Dis. 17, 541-551 (2009).

123 Fukuyama R, Izumoto T, Fushiki S. The cerebrospinal fluid level of glial fibrillary acidic protein is increased in cerebrospinal fluid from Alzheimer's disease patients and correlates with severity of dementia. Eur. Neurol. 46, 35-38 (2001).

124 Madeddu R, Farace C, Tolu P et al. Cytoskeletal proteins in the cerebrospinal fluid as biomarker of multiple sclerosis. Neurol. Sci. doi:10.1007/s10072-012-0974-4 (2012) (Epub ahead of print).

125 Brouns R, De Vil B, Cras P, De Surgeloose D, Mariën P, De Deyn P. Neurobiochemical markers of brain damage in cerebrospinal fluid of acute ischemic stroke patients. Clin. Chem. 56, 451-458 (2010).

126 Petzold A, Michel P, Stock M, Schluep M. Glial and axonal body fluid biomarkers are related to infarct volume, severity, and outcome. J. Stroke Cerebrovasc. Dis. 17, 196-203 (2008).

127 Aurell A, Rosengren L, Karlsson B, Olsson J, Zbornikova V, Haglid K. Determination of S-100 and glial fibrillary acidic protein concentrations in cerebrospinal fluid after brain infarction. Stroke 22, 1254-1258 (1991).

128 Fraser D, Close T, Rose K et al. Severe traumatic brain injury in children elevates glial fibrillary acidic protein in cerebrospinal fluid and serum. Pediatr. Crit. Care Med. 12, 319-324 (2011).

129 Czeiter E, Mondello S, Kovacs N et al. Brain injury biomarkers may improve the predictive power of the IMPACT Outcome Calculator. J. Neurotrauma 29(9), 1770-1778 (2012).

130 Neselius S, Brisby H, Theodorsson A, Blennow K, Zetterberg H, Marcusson J. CSF-biomarkers in Olympic boxing: diagnosis and effects of repetitive head trauma. PLoS One 7, e33606 (2012).

131 Ling H, Recklies A. The chitinase 3-like protein human cartilage glycoprotein 39 inhibits cellular responses to the 
inflammatory cytokines interleukin-1 and tumour necrosis factor-alpha. Biochem. J. 380, 651-659 (2004).

132 Bonneh-Barkay D, Wang G, Starkey A, Hamilton R, Wiley C. In vivo CHI3L1 (YKL-40) expression in astrocytes in acute and chronic neurological diseases. J. Neuroinflammation 7, 34 (2010).

133 Bonneh-Barkay D, Bissel S, Kofler J, Starkey A, Wang G, Wiley C. Astrocyte and macrophage regulation of YKL-40 expression and cellular response in neuroinflammation. Brain Pathol. 22(4), 530-546, (2012).

134 Chiasserini D, Parnetti L, Andreasson U et al. CSF levels of heart fatty acid binding protein are altered during early phases of Alzheimer's disease. J. Alzheimers Dis. 4, 1281-1288 (2010).

135 Steppan C, Bailey S, Bhat S et al. The hormone resistin links obesity to diabetes. Nature 409, 307-312 (2001).

136 Asakawa A, Inui A, Yuzuriha $\mathrm{H}$ et al. Characterization of the effects of pancreatic polypeptide in the regulation of energy balance. Gastroenterology 124, 1325-1336 (2003).

137 Jhamandas J, Li Z, Westaway D, Yang J, Jassar S, Mactavish D. Actions of $\beta$-amyloid protein on human neurons are expressed through the amylin receptor. Am. J. Pathol. 178, 140-149 (2011).

138 Vlassenko A, Vaishnavi S, Couture L et al. Spatial correlation between brain aerobic glycolysis and amyloid- $\beta$ (A $\beta$ ) deposition. Proc. Natl Acad. Sci. USA 107, 17763-17767 (2010).

139 Shuvaev V, Laffont I, Serot J, Fujii J, Taniguchi N, Siest G. Increased protein glycation in cerebrospinal fluid of Alzheimer's disease. Neurobiol. Aging 22, 397-402 (2001).

140 Ahmed N, Ahmed U, Thornalley P, Hager K, Fleischer G, Münch G. Protein glycation, oxidation and nitration adduct residues and free adducts of cerebrospinal fluid in Alzheimer's disease and link to cognitive impairment. J. Neurochem. 92, 55-63 (2005).

141 Montine T, Quinn J, Kaye J, Morrow J. $\mathrm{F}(2)$-isoprostanes as biomarkers of late-onset Alzheimer's disease. J. Mol. Neurosci. 1, 114-119 (2007).

142 Brys M, Pirraglia E, Rich K et al. Prediction and longitudinal study of CSF biomarkers in mild cognitive impairment. Neurobiol. Aging 30, 682-690 (2009).

143 De Leon M, Mosconi L, Li J et al. Longitudinal CSF isoprostane and MRI atrophy in the progression to AD. J. Neurol. 254, 1666-1675 (2007).

144 Ringman J, Younkin S, Pratico D et al. Biochemical markers in persons with preclinical familial Alzheimer disease. Neurology 71, 85-92 (2008).

145 Galasko D, Peskind E, Clark C et al. Antioxidants for Alzheimer disease. A randomized clinical trial with cerebrospinal fluid biomarker measures. Arch. Neurol. 69(7), 836-841 (2012).

-1. Decreased CSF F2-isoprostanes suggests that antioxidant therapy in a large clinical trial reduces brain oxidative stress.

146 Nunomura A, Tamaoki T, Motohashi N et al. The earliest stage of cognitive impairment in transition from normal aging to Alzheimer disease is marked by prominent RNA oxidation in vulnerable neurons. J. Neuropathol. Exp. Neurol. 71, 233-241 (2012).

147 Abe T, Tohgi H, Isobe C, Murata T, Sato C. Remarkable increase in the concentration of 8-hydroxyguanosine in cerebrospinal fluid from patients with Alzheimer's disease. J. Neurosci. Res. 3, 447-450 (2002).

148 Abe T, Isobe C, Murata T, Sato C, Tohgi H. Alteration of 8-hydroxyguanosine concentrations in the cerebrospinal fluid and serum from patients with Parkinson's disease. Neurosci. Lett. 336, 105-108 (2003).

149 Kikuchi A, Takeda A, Onodera H et al. Systemic increase of oxidative nucleic acid damage in Parkinson's disease and multiple system atrophy. Neurobiol. Dis. 9, 244-248 (2002).

150 Nunomura A, Hofer T, Moreira P, Castellani $\mathrm{R}$, Smith M, Perry G. RNA oxidation in Alzheimer disease and related neurodegenerative disorders. Acta Neuropathol. 118, 151-166 (2009).

151 Kaddurah-Daouk R, Kristal B, Weinshilboum R. Metabolomics: a global biochemical approach to drug response and disease. Annu. Rev. Pharmacol. Toxicol. 48, 653-683 (2008).

152 Kaiser E, Schoenknecht P, Kassner S, Hildebrandt W, Kinscherf R, Schroeder J. Cerebrospinal fluid concentrations of functionally important amino acids and metabolic compounds in patients with mild cognitive impairment and Alzheimer's disease. Neurodegener. Dis. 7, 251-259 (2010).

153 Czech C, Berndt P, Busch K et al. Metabolite profiling of Alzheimer's disease cerebrospinal fluid. PLoS One 2, e31501 (2012).

154 Mendell J, Olson E. MicroRNAs in stress signaling and human disease. Cell 148, 1172-1187 (2012).

155 Huntzinger E, Izaurralde E. Gene silencing by microRNAs: contributions of translational repression and mRNA decay. Nat. Rev. Genet. 12, 99-110 (2011).
156 Small E, Olson E. Pervasive roles of microRNAs in cardiovascular biology. Nature 469, 336-342 (2011).

157 Weber J, Baxter D, Zhang S et al. The microRNA spectrum in 12 body fluids. Clin. Chem. 56, 1733-1741 (2010).

158 Baraniskin A, Kuhnhenn J, Schlegel U et al. Identification of microRNAs in the cerebrospinal fluid as marker for primary diffuse large B-cell lymphoma of the central nervous system. Blood 117, 3140-3146 (2011).

159 Gallego J, Gordon M, Claycomb K, Bhatt M, Lencz T, Malhotra A. In vivo microRNA detection and quantitation in cerebrospinal fluid. J. Mol. Neurosci. 47(2), 243-248 (2012).

160 Teplyuk N, Mollenhauer B, Gabriely G et al. MicroRNAs in cerebrospinal fluid identify glioblastoma and metastatic brain cancers and reflect disease activity. Neuro. Oncol. 14(6), 689-700 (2012).

161 Cogswell J, Ward J, Taylor I et al. Identification of miRNA changes in Alzheimer's disease brain and CSF yields putative biomarkers and insights into disease pathways. J. Alzheimers Dis. 14, 27-41 (2008).

162 Bateman R, Wen G, Morris J, Holtzman D. Fluctuations of CSF amyloid-beta levels: implications for a diagnostic and therapeutic biomarker. Neurology 68, 666-669 (2007).

163 Moghekar A, Goh J, Li M, Albert M, O’Brien R. Cerebrospinal fluid $A \beta$ and tau level fluctuation in an older clinical cohort. Arch. Neurol. 69, 246-250 (2012).

164 Verwey N, van der Flier W, Blennow Ket al. A worldwide multicentre comparison of assays for cerebrospinal fluid biomarkers in Alzheimer's disease. Ann. Clin. Biochem. 46, 235-240 (2009).

165 Bjerke M, Portelius E, Minthon L et al. Confounding factors influencing amyloid beta concentration in cerebrospinal fluid. Int. J. Alzheimers Dis. 2010, 986310 (2010).

166 Teunissen C, Verwey N, Kester M, van Uffelen K, Blankenstein M. Standardization of assay procedures for analysis of the CSF biomarkers amyloid $\beta(1-42)$, tau, and phosphorylated tau in Alzheimer's disease: report of an international workshop. Int. J. Alzheimers Dis. 2010, 635053 (2010).

167 Mattsson N, Andreasson U, Persson S et al. The Alzheimer's Association external quality control program for cerebrospinal fluid biomarkers. Alzheimers Dement. 7, 386-395 (2011).

168 Fodero-Tavoletti M, Okamura N, Furumoto $S$ et al. ${ }^{18} \mathrm{~F}-\mathrm{THK} 523$. A novel in vivo tau imaging ligand for Alzheimer's disease. Brain 134, 1089-1100 (2011). 
169 De Leon MJ, Desanti S, Zinkowski R et al. Longitudinal CSF and MRI biomarkers improve the diagnosis of mild cognitive impairment. Neurobiol. Aging 27, 394-401 (2006).

170 Hampel H, Burger K, Pruessner J et al. Correlation of cerebrospinal fluid levels of tau protein phosphorylated at threonine 231 with rates of hippocampal atrophy in Alzheimer disease. Arch. Neurol. 62, 770-773 (2005).

171 Lo R, Hubbard A, Shaw L et al. Longitudinal change of biomarkers in cognitive decline. Arch. Neurol. 68, 1257-1266 (2011).

172 Britschgi M, Rufibach K, Huang S et al. Modeling of pathological traits in Alzheimer's disease based on systemic extracellular signaling proteome. Mol. Cell. Proteomics 10, M111.008862 (2011).
173 Vemuri P, Wiste H, Weigand S et al. MRI and CSF biomarkers in normal, MCI and $\mathrm{AD}$ subjects. Diagnostic discrimination and cognitive correlations. Neurology 73, 287-293 (2009).

174 Zhang D, Wang Y, Zhou L, Yuan H, Shen D, Alzheimer's disease neuroimaging initiative: multimodal classification of Alzheimer's disease and mild cognitive impairment. Neuroimage 55, 856-867 (2011).

175 Vos S, van Rossum I, Burns L et al. Test sequence of CSF and MRI biomarkers for prediction of $\mathrm{AD}$ in subjects with MCI. Neurobiol. Aging doi:10.1016/j. neurobiolaging.2011.12.017 (2012) (Epub ahead of print).

176 Hampel H, Wilcock G, Andrieu S et al. Biomarkers for Alzheimer's disease therapeutic trials. Prog. Neurobiol. 95 , 579-593 (2011).

177 McKhann G, Knopman D, Chertkow H et al. The diagnosis of dementia due to Alzheimer's disease: recommendations from the National Institute on Aging and the Alzheimer's Association Workgroup. Alzheimers Dement. 7, 263-269 (2011).

\section{Websites}

201 Alzheimer's Association. 2011 Alzheimer's disease facts and figures.

www.alz.org/national/documents/Facts_ Figures_2011.pdf

202 Alzheimer Research Forum. Biomarkers. www.alzforum.org/res/com/biomarkers/ default.asp 